\title{
Occlusion Gesture Recognition Based on Improved SSD
}

\author{
Shangchun Liao ${ }^{\mathrm{a}}$, Gongfa Li ${ }^{\mathrm{a}, \mathrm{c}}$, Hao Wu ${ }^{\mathrm{a}}$, Du Jiang ${ }^{\mathrm{a}}$, Ying Liu', Juntong Yuna, Yibo \\ Liu $^{\mathrm{a}}$ and Dalin Zhou ${ }^{\mathrm{d}}$ \\ ${ }^{a}$ Key Laboratory of Metallurgical Equipment and Control Technology of Ministry of \\ Education, Wuhan University of Science and Technology, Wuhan 430081, China \\ ${ }^{\mathrm{b}}$ Research Center for Biomimetic Robot and Intelligent Measurement and Control, \\ Wuhan University of Science and Technology, Wuhan 430081, China \\ ${ }^{\mathrm{c}}$ Hubei Key Laboratory of Mechanical Transmission and Manufacturing Engineering, \\ Wuhan University of Science and Technology, Wuhan 430081, China \\ ${ }^{d}$ School of Computing, University of Portsmouth, Portsmouth PO1 3HE, UK
}

\begin{abstract}
: gesture recognition has always been a research hotspot in the field of human-computer interaction. Its purpose is to realize the natural interaction with the machine by recognizing the semantics expressed by gesture. In the process of gesture recognition, the occlusion of gesture is an inevitable problem. In the process of gesture recognition, some or even all of the gesture features will be lost due to the occlusion of the gesture, resulting in the wrong recognition or even unrecognizability of the gesture. Therefore, it is of great significance to study gesture recognition under occlusion. The SSD algorithm is analyzed, and the front-end network is compared. Mobilenets is selected as the front-end network, and the Mobilenets-SSD network is improved. In tensorflow environment, based on the improved network model, the self-occlusion gesture and object occluding gesture are trained in color map, depth map and color and depth fusion respectively, and the recognition models of self-occlusion gesture and object occluding gesture are obtained. And the various models are compared and analyzed.
\end{abstract}

Key words: gesture recognition, occlusion, SSD, human computer interaction

\section{Introduction}

The realization of gesture recognition brings a new immersive experience for human-computer interaction. Due to the nature and simplicity of interaction, gesture recognition technology based on vision has been greatly developed in recent years. However, due to the variability of external environment and the flexibility of hand posture, the current gesture recognition technology cannot replace the existing interaction equipment, so it is imperative to further study gesture recognition technology. Color image can provide clear appearance, texture and other features, depth image can overcome the sensitivity of light changes, has good robustness to light, and can provide clear shape and edge information, and in the normal human-computer interaction process, gesture will have the situation of blocking. Therefore, in the case of occlusion, how to use depth and color information to correctly recognize the occlusion gesture is the difficulty in the current human-computer interaction technology ${ }^{[1]}$.

In order to solve the occlusion problem in the process of gesture recognition, this study uses the improved SSD algorithm for occlusion gesture recognition, and collects 
a large number of object occlusion gesture and self-occlusion gesture data samples to establish the gesture database for research; using the self-established database, the improved SSD algorithm is trained by color map, depth map, color map and depth map fusion respectively, and three kinds of packages are obtained Occlusion gesture recognition models including self-occlusion and object occlusion are proposed, and the recognition results of the three models are analyzed and compared.

The rest of this paper is organized as follows. Section 2 is about the related research in this field. Section 3 introduces the improvement of SSD network. Section 4 describes collection of gesture database and preprocessing of gesture data. Section 5 carries on the model training and the recognition result analysis and the contrast. Section 6 draws the conclusion and gives the discussion of future work.

\section{Related Work}

For static gesture recognition, Singha et al extract the hand image from the original image by image processing technology such as skin color filtering and edge detection $^{[2]}$, then use the KLT (Karhunen-Loeve Transform) algorithm for feature extraction, and finally effectively classify and recognize the gesture. Kuznetsova and others first transform the depth map of gesture into point cloud, then extract the descriptor of shape function set (ESF) ${ }^{[3]}$, classify gesture through multi-layer random forest, reduce training time and achieve better recognition effect. Maqueda and others proposed a vs-lbp video descriptor for dynamic gesture recognition ${ }^{[4]}$, which can provide more abundant spatiotemporal information and has controllable computing cost. Priyal et al extracted the Krawtchouk feature of the contour to represent the static gesture $^{[5]}$, and used the minimum distance classifier to classify. This method has strong robustness to similarity transformation and perspective deformation. In gesture recognition, the most direct and simple method is to use the unique shape and contour of human hand in the human-computer interaction environment. The method based on shape matching matches the pre constructed shape model and observed shape features. Belongie and others proposed the method based on shape detection and matching for gesture recognition ${ }^{[6]}$. They introduced context descriptor method and context description method This method is used to detect similar shapes in different images and achieves better results.

With the continuous development of convolutional neural network, gesture recognition based on deep learning has achieved good results. Proposed a typical gesture recognition method based on LFMCW (linear frequency modulated continuous wave) radar range-Doppler (RD) information and convolutional neural network. Using LFMCW radar echo, the two-dimensional Rd image data of gesture target is obtained, and the RD image amplitude matrix is used as CNN input sample to achieve effective recognition of gesture action. For robust gesture recognition in noisy background and unconstrained environmental factors, Dadashzadeh A and others proposed a new two-stage convolution neural network, called HGR net, is proposed ${ }^{[7]}$. In the first stage, accurate pixel level semantic segmentation is carried out to determine the hand area, and in the second stage, gesture recognition is carried out. The phased network structure is based on the combination of full convolution residual network and spatial pyramid pooling. In the recognition stage, the dual stream CNN 
network is used to fuse the RGB information and the segmented image information, and their depth representation is combined into a new full connection layer before classification. The test results on the public dataset show that the method achieves high recognition rate in image segmentation and static gesture recognition.

In human-computer interaction, gesture recognition under normal conditions will have intermittent occluded gestures. How to recognize gesture under occluded conditions is a research difficulty ${ }^{[8]}$. Rangesh $\mathrm{A}$ and others proposed a gesture detection framework using monocular camera under occlusion ${ }^{[9]}$. In order to prove the robustness of the detection framework, the detection framework is evaluated on a challenging and occluded natural driving data set. The figures of the driver's hand movement in the data set are reliable. In addition, the framework also encodes and learns the complex gesture interaction actions offline, and makes the best choice in data association. Mueller $\mathrm{F}$ and others proposed a method of occlusion hand pose estimation based on RGB-D camera, using two CNN networks to locate the position of hand and $3 \mathrm{D}$ joint regression of hand ${ }^{[10]}$. In the case of occlusion or no occlusion, $\mathrm{CNN}$ is used to estimate the two-dimensional position of the hand center, then input the corresponding depth value, and input it to the second CNN network at the same time, so as to realize the regression of the three-dimensional hand joint position, so as to realize the gesture recognition under occlusion. In order to solve the problem of self-occlusion of hand gestures, Poon G and others proposed a method based on multi view feature fusion to recognize hand gestures ${ }^{[11]}$. A multi camera system is constructed, which uses multiple cameras with different viewing angles to obtain multi view hand potential data, uses its shape and color features to train the hand gesture data set, uses the method of weighted fusion, uses different classifiers to predict the results, and the experimental results show that the multi view recognition effect is better than the single view.

\section{Improved SSD network}

\subsection{SSD network framework structure and principle}

Based on the feedforward convolution network, SSD generates a fixed size frame set and scores the detailed target categories in the frame. Then, the final detection results are generated by the non maximum suppression step. As shown in Figure 1, a default box with different aspect ratio is generated at each pixel of the feature map, and then each default box is regressed and classified. In figure (a), the SSD network only needs a real label of each target during the input image and training period, and evaluates a small set of default boxes with different aspect ratios at each location in several feature maps on different scales (As shown in $8 \times 8$ and $4 \times 4$ in figures (b) and (c)) by convolution. For all default boxes, the shape offset and confidence of all object categories $(c 1, c 2, \ldots, c p)$ are predicted. During training, these default boxes are first matched to the real label boxes ${ }^{[12-15]}$. 


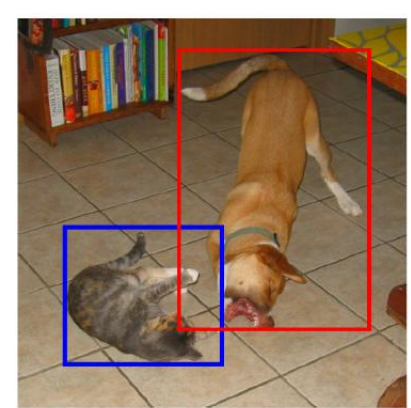

(a) Image with GT boxes

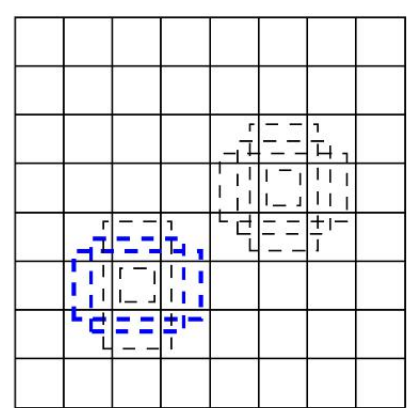

(b) $8 \times 8$ feature map

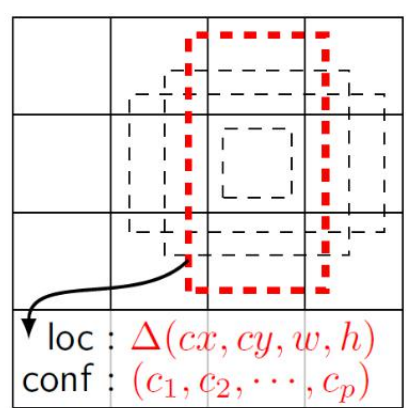

(c) $4 \times 4$ feature map

Figure 1. SSD default box

SSD network adds several feature layers at the end of the basic network. These feature layers predict the default frame of different scales and aspect ratio and their correlation confidence. A set of default bounding boxes is associated with each feature mapping unit for use with multiple feature maps at the top of the network. The default box is tiled by convolution, so that the position of each default box relative to its corresponding cell is fixed ${ }^{[15-17]}$. In each feature mapping cell, the offset from the default box shape in the cell is predicted, as well as the rating of each category that indicates the detailed category in which each box exists.

\subsection{Mobilenets-SSD network}

Mobilenets is a model for mobile devices and embedded visual applications. It is based on streamline structure and uses Depthwise separable convolution to build lightweight depth neural network. Mobilenets decomposes the convolution kernel mainly through the depth separable convolution, and decomposes the standard convolution into a depth convolution and a $1 * 1$ point convolution ${ }^{[18]}$. For this deep convolution, a single filter can be applied to each input channel, and then the point convolution applies $1 * 1$ convolution is combined with the output of the deep convolution ${ }^{[19]}$. In addition, two super parameters, width multiplier and resolution multiplier, are introduced into the network. These two parameters make the network model reach a balance in terms of delay and accuracy ${ }^{[20]}$. The width multiplier is mainly used to reduce the number of input and output channels, and the resolution multiplier is used to reduce the size of the input and output characteristic map.

(1) Standard convolution

The standard convolution is to accept the input as $D_{F} \times D_{F} \times M$ feature map $F$, and get the output as $D_{G} \times D_{G} \times N$ feature map $G, D_{F}$ is the width and height of a square feature map with input, $M$ is the number of input channels; $D_{G}$ is the width and height of a square feature map with output, and $N$ is the number of output channels. The calculation of standard convolution is $D_{K} \cdot D_{K} \cdot M \cdot N \cdot D_{F} \cdot D_{F}$, where $D_{K} \times D_{K}$ is the size of the convolution kernel $K, M 、 N$ are the number of input channels and output channels respectively ${ }^{[21-23]}$. The output of the standard convolution feature map is calculated as: 


$$
G_{k, l, n}=\sum_{i, j, m} K_{i, j, m, n} \cdot F_{k+i-1, l+j-1, m}
$$

In the formula:

$k 、 l$ are the height and width of the output characteristics respectively;

$i 、 j$ are the height and width of convolution kernel;

$m 、 n$ are the number of input and output channels respectively.

(2) Depth separable convolution

Depth separable convolution is divided into two steps: depth convolution and point convolution. Each convolution kernel is used for each input channel by depth convolution, and then point convolution of $1 \times 1$ is used to combine the output of depth convolution ${ }^{[24-25]}$.

The single filter of each input channel of deep convolution can be as follows (2):

$$
\hat{G}_{k, l, m}=\sum_{i, j} \hat{K}_{i, j, m} \cdot F_{k+i-1, l+j-1, m}
$$

In depth convolution, the size of convolution kernel is $D_{K} \cdot D_{K} \cdot M$, and the calculation amount of depth convolution is $D_{K} \cdot D_{K} \cdot M \cdot D_{F} \cdot D_{F}$; In the calculation of point convolution, the size of convolution kernel is $1 \times 1 \times M \times N$, the calculation amount of point convolution is $M \cdot N \cdot D_{F} \cdot D_{F}$, the calculation amount of depth separable convolution is $D_{K} \cdot D_{K} \cdot M \cdot D_{F} \cdot D_{F}+M \cdot N \cdot D_{F} \cdot D_{F}$, and the calculation amount ratio of standard convolution and depth separable convolution is as shown in equation (3):

$$
\frac{D_{K} \cdot D_{K} \cdot M \cdot D_{F} \cdot D_{F}+M \cdot N \cdot D_{F} \cdot D_{F}}{D_{K} \cdot D_{K} \cdot M \cdot N \cdot D_{F} \cdot D_{F}}=\frac{1}{N}+\frac{1}{D_{K}^{2}}
$$

Mobilenets uses the depth separable convolution of $3 \times 3$, so the computation is 8-9 times less than the standard convolution ${ }^{[26]}$.

(3) Width multiplier and resolution multiplier

Width multiplier is used to reduce the number of channels, which is represented by $\alpha$. the value range is generally $0<\alpha \leq 1$, that is, when the original number of input and output channels is $m 、 n$, the channel numbers are $\alpha m 、 \alpha n$ respectively after introducing the super parameter width multiplier. When the width multiplier is introduced [27], the computation amount of depth separable convolution is $D_{K} \cdot D_{K} \cdot \alpha M \cdot D_{F} \cdot D_{F}+\alpha M \cdot \alpha N \cdot D_{F} \cdot D_{F}$.

Resolution multiplier is used to reduce the size of the feature map in proportion, which is represented by $\rho$. Therefore, after the introduction of resolution parameters, 
the total calculation amount of the depth separation convolution network is $D_{K} \cdot D_{K} \cdot \alpha M \cdot \rho D_{F} \cdot \rho D_{F}+\alpha M \cdot \alpha N \cdot \rho D_{F} \cdot \rho D_{F}$, and the calculation amount that can be reduced is $\rho^{2}$.

As shown in Figure 2, the network detection framework of Mobilenets-SSD is to replace the front-end network vgg16 in SSD network with Mobilenet, which removes the global average pooling layer, full connection layer and sofamax layer of Mobilenet network, and then connects the back-end detection network of SSD to form the Mobilenets-SSD network ${ }^{[28]}$. Because the depth of the front-end network of Mobilenets SSD network is deeper than that of SSD network, the depth of the whole model is greater than that of SSD network. From the perspective of SSD back-end detection network, both Mobilenets-SSD and SSD network use feature extraction from six scale feature map for detection. Because Mobilenets-SSD network uses deep separable convolution, the resolution of feature map of back-end detection network is only half of SSD network, so the network has less computation and computational complexity ${ }^{[29]}$.

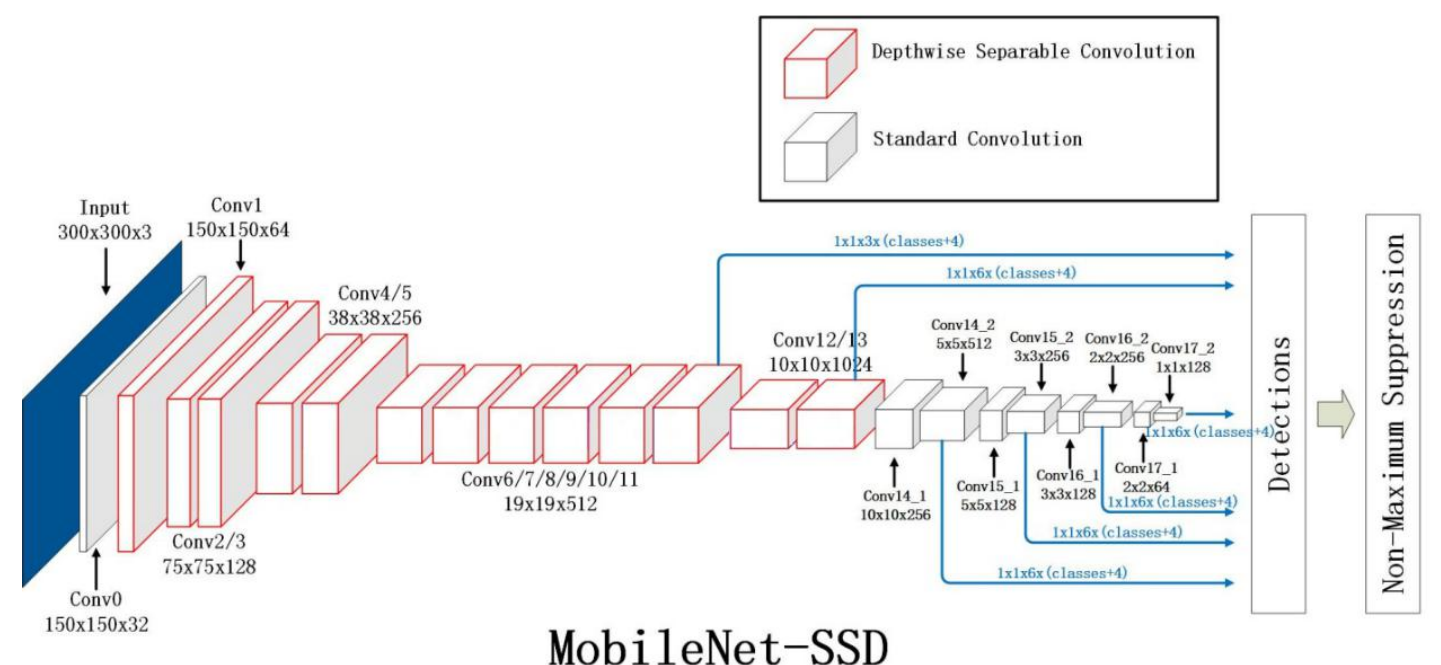

Figure 2. MobileNets-SSD network structure

\subsection{Improved MobileNets-SSD network}

The depth neural network is composed of $n$ layer structure. The $i$ layer is represented by $L_{i}$. the output dimension of its activation layer is $h_{i} \times w_{i} \times d_{i}$, that is, it contains $d_{i}$ channels, and each channel has $h_{i} \times w_{i}$ pixels. Generally speaking, it is considered that the set of activation layers forms a "manifold of interest", which is embedded in the low-dimensional subspace through the manifold of interest in the neural network. In other words, when we look at all the individual channel pixels in the deep convolution, the information exists in some kinds of maniford in some encoding way and then is embedded in the low-dimensional subspace. Roughly speaking, we can reduce the dimension of the activation space by reducing the layer dimension. Mobilenets network uses this point to reduce the dimension of the 
activation space by the wide high multiplier until the manifold of interest spans the whole space ${ }^{[30-32]}$. But when the depth neural network makes nonlinear transformation to each coordinate, this method fails. For example, when using the ReLU activation function to make nonlinear changes, a line in one-dimensional space will generate a ray, while in $R^{n}$ space, a piecewise linear curve with $\mathrm{n}$ joints will usually be generated. Generally speaking, when the activation function is ReLU, the function of the neural network is equivalent to a linear transformation in the non-zero part of the input domain. As shown in Figure 3, the ReLU transformation of a low dimensional manifold embedded in a high dimensional space. The input is a two-dimensional helix. Firstly, the input is mapped to the $\mathrm{n}$ dimension through the matrix, then to the two-dimensional space through the ReLU function, and finally to the two-dimensional space through the transposition matrix of the matrix. When $n=2$ or $n=3$, we can see that the center point collapses, the information loss is very serious, and when $n=15$ or higher, the information is relatively complete. Because when the number of channels is small, the information is concentrated in these channels. If some values are less than 0 , they will be lost by the ReLU activation function. However, when the number of channels is large, the information is scattered and redundant, so the value regressed to 0 through ReLU activation may not affect the storage of too much information. This shows that the nonlinear transformation in low dimensional space will lose a lot of information, which may not exist when the latitude is high.
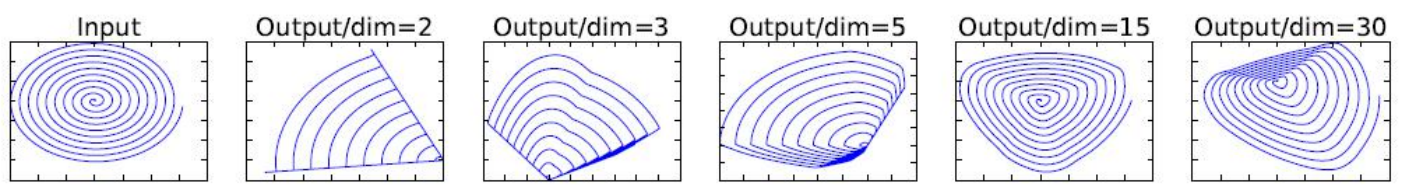

Figure 3. ReLU transformation of embedded low dimensional manifold in high dimensional space

Therefore, by improving the network structure to avoid the loss of feature information in the low-dimensional space, the improved network is shown in Figure 4. By replacing the activation function ReLU after point convolution (PW) in the depth scalable convolution, the dimension of the low-dimensional feature space will not be reduced again, thus ensuring the features in the low-dimensional space ${ }^{[33-35]}$.

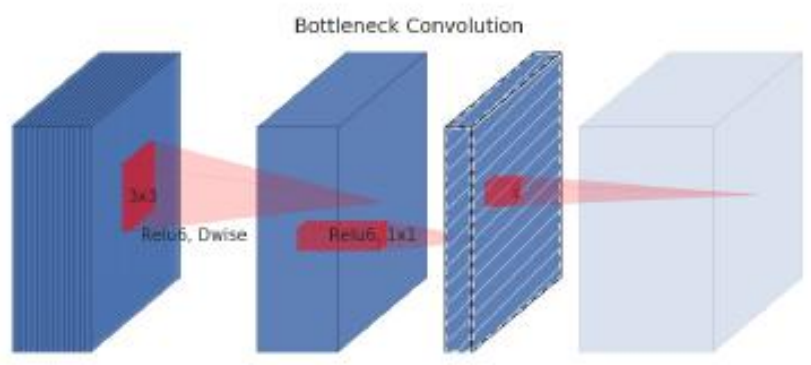

Figure 4. Linear robot structure 
Because the depth separable convolution itself has no ability to change the number of channels, the number of channels given by the previous layer can only be output. If the number of channels given by the previous layer is small, the depth separable convolution can only extract features in low dimensional space, so the effect is not good. In order to solve this problem, it is necessary to increase the dimension of the channel number before the depth separable convolution, and use the point convolution in the residual structure to increase the dimension of the channel number. Therefore, before the depth separable convolution (DW), the point convolution (PW) is used to increase the dimension of channel number, and the dimension increasing coefficient is defined as $t$, so no matter what the input channel number $C_{i n}$ is before the dimension increasing, DW works in a relatively higher dimension $t C_{i n}$ after PW dimension increasing, which improves the feature extraction effect ${ }^{[36-38]}$. The comparison between the improved network structure and the mobilenets structure is shown in Figure 5.
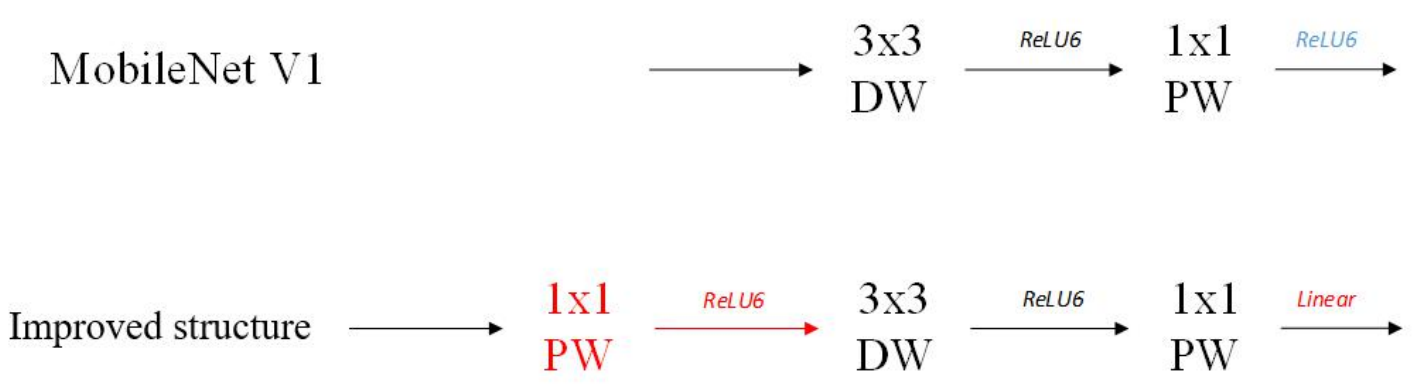

Figure 5. Improved mobilenets architecture

The improved MobileNets-SSD network improves the efficiency of feature extraction by improving the network structure of Mobilenet. The improved network structure of MobileNets-SSD and the network structure based on MobileNets-SSD are basically unchanged. The front-end feature extraction network Mobilenet is improved. The back-end network still uses the back-end detection network of SSD to extract features from six different scales, so as to improve the efficiency of feature extraction The detection effect of the whole network on low dimensional channels.

\section{Gesture database and gesture preprocessing}

\section{1 gesture database}

In this paper, we study occlusion gesture recognition based on convolutional neural network. Gesture is self-occlusion gesture and object occlusion gesture respectively. So when we collect gesture, we need to collect self-occlusion gesture and object occlusion gesture respectively, and build its database. In this paper, Kinect is used to collect the color data and depth data of occlusion gesture. The color data contains color, texture and other features. The depth information of objects in the depth data does not contain color and other information, and the depth data is not sensitive to light and environmental changes. In this paper, the hand gesture recognition model is 
established based on color information, depth information and depth and color information fusion respectively ${ }^{[39-41]}$. Therefore, when collecting images, we need to pay attention to the collection of hand gesture data under different background and light conditions, so that the hand gesture recognition model has good robustness. In this paper, four kinds of self-occlusion gesture data are collected, and the color map and depth map of four kinds of gesture are defined as shown in Figure 6. Considering the difference of gesture, four samples of gesture were collected for each gesture. Under different background, 9600 color images and 9600 depth images were obtained respectively, 8000 of which were training sets and 1600 were test sets. The color database and depth database of self-occlusion gesture were established.

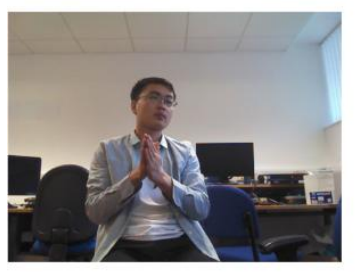

Gesture l

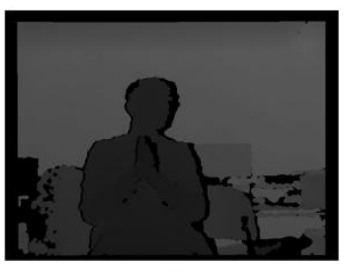

Gesture1

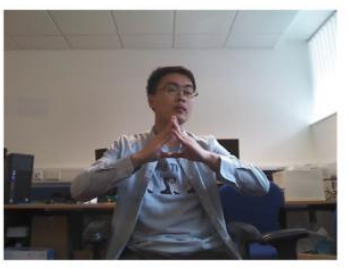

Gesture2

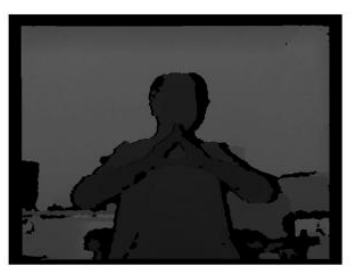

Gesture2

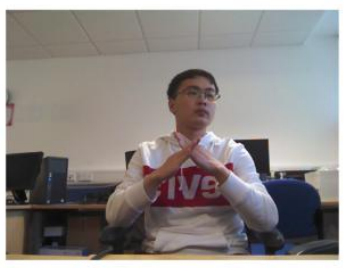

Gesture3

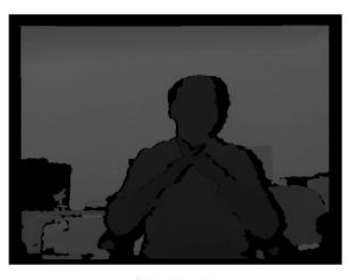

Gesture3

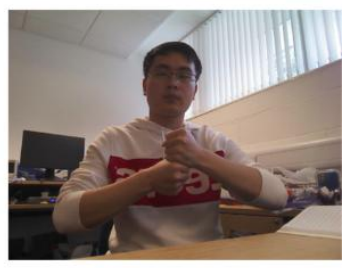

Gesture4

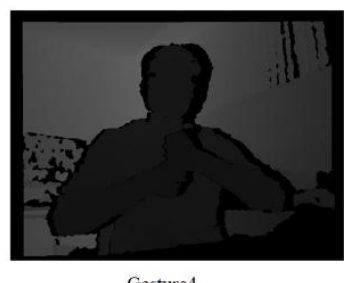

Gesture4

Figure 6. Self-occlusion gesture

The gesture of object occlusion can be recognized under the condition of occlusion or not, the occlusion rate of the whole hand gesture is $0-20 \%$ in this study, that is, the occlusion area does not exceed $20 \%$ of the whole hand area. When collecting gesture data, a large number of unobstructed gestures (occlusion rate $0 \%$ ) and occluded gestures with occlusion rate less than $20 \%$ are collected to establish occluded gesture database $^{[42-43]}$. The five defined occlusion gestures are the five gestures with numbers 1-5 in ASL sign language library, as shown in Figure 7, which are color and depth maps of the five occlusion gestures. Five kinds of gestures in ASL gesture database are selected to obtain 10625 color and depth images of five kinds of gestures in occluded and unobstructed conditions, among which 8500 constitute the training set and 2125 constitute the test set. The color database and depth database of object occluded gestures are established respectively. 


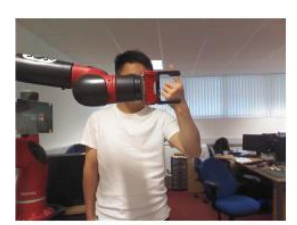

Hand1

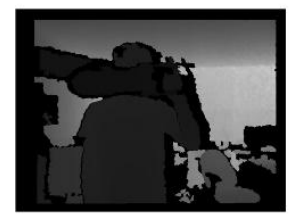

Hand1

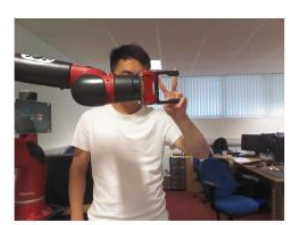

Hand2

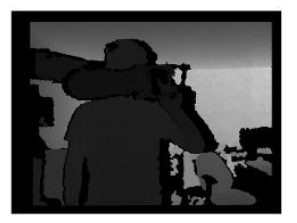

Hand2

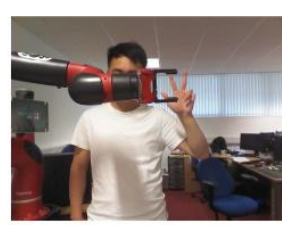

Hand3

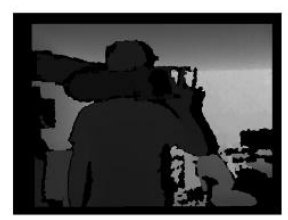

Hand3

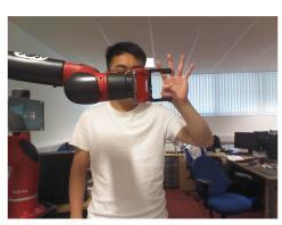

Hand4

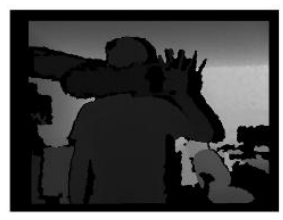

Hand4

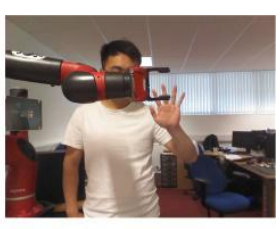

Hand5

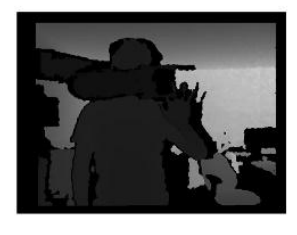

Hand5

Figure 7. Gesture of object occlusion

\subsection{Data preprocessing}

The color gesture database of two hand gesture and one hand gesture is annotated by labelimg. The four gestures selected from the two hand gesture database are marked as gesture1, gesture2, gesture 3 and gesture4. The gestures in the one hand gesture database represent 1-5 gestures selected from the ASL gesture database, which are respectively marked as hand1, hand2, hand3, hand4 and hand5. After labeling the gesture database, each picture will generate an XML file, which contains the file name, picture size, category information and coordinate information of the gesture $^{[44]}$.

After the data is annotated, the XML file with annotation information and the image data are transformed into the file for training under the Tensorflow deep learning framework, and the file format is TFRecord. The training set and test set are carried out in the above steps respectively, so far the data preprocessing is completed, and the model training files under the color map are obtained. Kinect's color map and depth map are aligned, so the manual annotation file of color map can be directly used in depth map, and together with depth map, it can be transformed into TFRecord file that can be used for training under Tensorflow framework, and the file that can be used for model training under depth map can be obtained ${ }^{[45-48]}$. In order to use both color image and depth image for gesture recognition, it is necessary to fuse the information of color image and depth image. The fusion mode of color information and depth information based on traditional neural network is generally divided into pre fusion, feature layer fusion, post fusion and full connection layer fusion. In this paper, the pre fusion method is adopted, and the color information and depth information are used for occlusion gesture recognition. Because the color map and depth map are aligned, the color map and its corresponding depth map are fused to get four channel images ${ }^{[49-51]}$, and then they are processed together with the label file to get the test set and training set of the fusion data of self-occlusion gesture and object occlusion gesture in Tensorflow framework.

\section{Experiment}

5.1 Hand gesture recognition model based on SSD network 
(1) Hand gesture recognition model based on color image

Call the training and testing configuration file, start to train the two hand gesture recognition model of color map. In the training process, gradient descent method is also used to train, and the batch_size is set to 32 , the initial learning rate is set to 0.004 , the learning rate of 30000 steps per iteration decreases to $95 \%$, and the number of iterations in the whole training process is 200000. Learning rate is an important parameter in deep learning. Choosing appropriate initial learning rate is the key to whether and when the model converges. Of course, the smaller the learning rate is, the easier it is to make the model converge, but the training time will be multiplied, so it is necessary to choose the appropriate initial learning rate ${ }^{[52-53]}$. After the parameters in the configuration file are correctly configured, the training process file will be generated from step 0 to step 200000 in the training process. After completing the whole training process, the model file which iterates to 200000 steps is transformed into $\mathrm{pb}$ model file and saved to get the two hand gesture recognition model under the color background.

(2) Hand gesture recognition model based on depth map

In order to compare the effect of hand gesture recognition with that of color background, the network structure and parameter configuration of hand gesture recognition model training based on depth map are the same, only the test set (train.record) and training set (test.record) change to the corresponding depth data set. The training method is the same as that in the color background, the gradient descent method is also used in the training, the batch_size is set to 32, the initial learning rate is 0.004 , and the learning rate of every 30000 steps decreases to $95 \%$. The training process has 200000 iterations in total. During the training process, training process files will be generated continuously. After the training, the training process files will also be converted into $\mathrm{pb}$ model files.

(3) Hand gesture recognition model based on color and depth features

In the data preprocessing, the test set and training set of the fusion data have been obtained. The model of hand gesture recognition based on color and depth fusion also adopts the improved Mobilenets-SSD network structure, so only the data set needs to be replaced by the test set and training set of color depth fusion in the training process. The training method is the same as that in color background and depth background, the gradient descent method is also used in training, the batch size is set to 32, the initial learning rate is 0.004 , and the learning rate of every 30000 steps decreases to $95 \%$. The training process has 200000 iterations in total. The training process files will be generated continuously during the training process. After the training, the training process files will also be converted into $\mathrm{pb}$ model files to obtain the two hand self-occlusion gesture recognition model based on color and depth fusion.

\subsection{Recognition model of occlusion gesture based on SSD network}

(1) Recognition model of occlusion gesture based on color image

The gesture recognition based on object occlusion also adopts the improved MobileNets-SSD network structure, which is basically the same as the configuration parameters of the two hand self-occlusion gesture recognition model. After the pre-processing of data, the data set of occlusion gesture in color background is 
obtained. The training set and test set are replaced by the training set and test set of occlusion gesture in color background. Because the same network architecture is used, the parameters in the configuration file are basically the same, the gradient descent method is also used in training, the batch_size is set to 32 , the initial learning rate is set to 0.004 , the learning rate of 30000 steps of each iteration is reduced to $95 \%$, and 200000 steps of the whole process are iterated. Five kinds of gestures are set for object occlusion gesture, and the information of five kinds of gestures are marked in the label file.

After completing the parameter setting of the configuration file, start to train the object occlusion gesture recognition model based on the color background. During the training process, the training process file will also be generated with the number of iteration steps. After the training, the training process file is transformed into a $\mathrm{pb}$ model file, and then an object occlusion gesture recognition model in color background is obtained.

(2) Recognition model of occlusion gesture based on depth map

In the training process, the system environment and network structure configuration are the same as that in the color background. Only the color data needs to be replaced by the depth data. The training of recognition model in depth background is the same as that in color background. In order to compare the recognition effect of color background and depth background, the gradient descent method is also used in training, the batch_size is set to 32 , the initial learning rate is set to 0.004 , the number of iteration steps is 200000, and the learning rate of each iteration 30000 steps decreases to $95 \%$.

After the completion of parameter configuration, start the training of occlusion gesture recognition based on the depth background. During the training process, the training process file will also be generated continuously, which will be converted into $\mathrm{pb}$ model file, so as to get the gesture recognition model based on object occlusion in the depth background ${ }^{[54-56]}$.

(3) An object occlusion gesture recognition model based on color and depth features

In the data preprocessing, through the pre fusion, the training set and test set of the fusion data of the object occlusion gesture are obtained. Because of the improved MobileNets-SSD network structure, only the data set needs to be replaced by the fusion data set. The training method is the same as that based on color map or depth map, the gradient descent method is also used in training, the batch size is set to 32, the initial learning rate is 0.004 , and the learning rate of every 30000 steps decreases to $95 \%$. The training process has 200000 iterations in total. The parameter settings are the same, which is more scientific when comparing the results. The training process files will be generated continuously during the training process. After the training, the training process files will be converted into $\mathrm{pb}$ model files, and the two hands self-occlusion gesture recognition model based on color and depth fusion will be obtained.

4.3 Analysis of experimental results of occlusion gesture

5.3.1 Results and analysis of hand gesture recognition model 


\section{(1) Learning rate}

Learning rate is an important super parameter in deep learning. It controls the speed of adjusting neural network weight based on gradient loss and the speed of updating parameters. If the learning rate is set too large, it will cause the loss function to explode and fluctuate around the minimum value without convergence; if the learning rate is set too small, it will cause the learning speed to slow down. Although the smaller learning rate can ensure that no local best can be missed, it will take more time to converge. The smaller learning rate is generally used for the time point after several rounds.

A larger learning rate and attenuation coefficient can be set at the beginning to make the loss function decrease at a faster speed at the beginning. With the increase of training times, the learning rate gradually decreases and the loss function converges at a slower speed. In this chapter, the initial learning rate is set to 0.004 , the learning rate attenuation coefficient is set to 0.95 , and the number of attenuation steps is 30000 . When iterating to 200000 times, the learning rate is 0.0029404 . The change curve of learning rate with the number of iterations in the whole training process is shown in Figure 8.

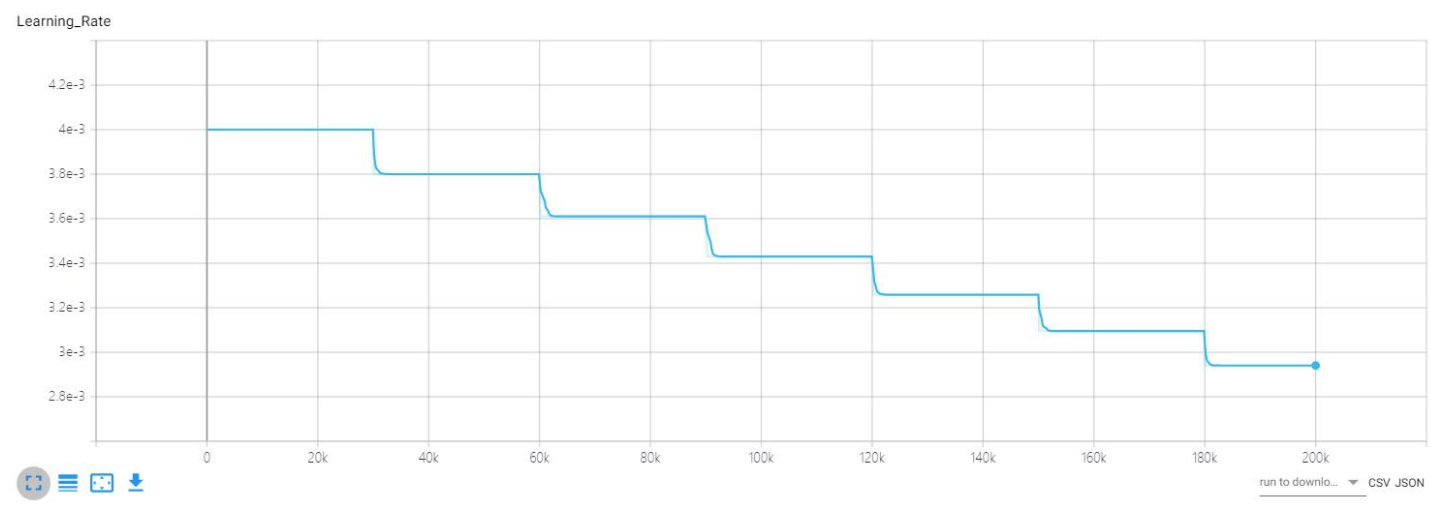

Figure 8. Change curve of learning rate

(2) Loss function

Loss function is used to measure the inconsistency between the predicted value and the real value of the model. It is a non negative real value function. The smaller the loss value of the function is, the closer the predicted value of the model is to the real value. The larger the loss function is, the greater the difference between the predicted value and the real value of the model is. The loss function of SSD network is equal to the weighted sum of the confidence loss and the position loss. In Figure 9, a), b) and C) are the curves of the classification loss, position loss and target loss of the whole model with the number of steps in succession. It can be seen from the figure that when the classification loss is iterated to about 1000 steps, the damage function decreases from 22.07 to near 1, and oscillates near 1, basically reaching convergence; when the location loss is iterated to about 2000 steps, the loss function rapidly decreases from 3.594 to near 0.1 , and oscillates back and forth nearby. When the objective loss function (the weighted sum of confidence loss and position loss) of the model is iterated to about 1000 steps, the loss function drops rapidly from 26.01 to the 
vicinity of 1 , and oscillates back and forth in the vicinity, basically reaching convergence.

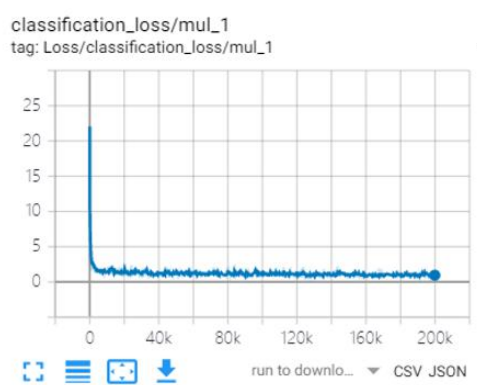

a) Classification loss

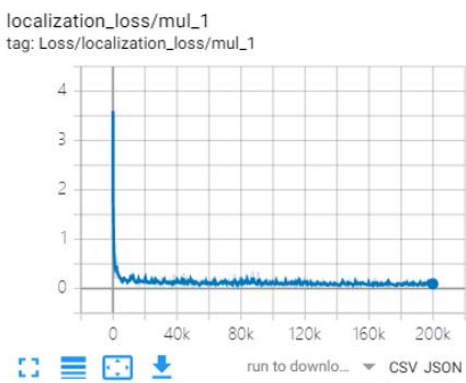

b) Localization loss

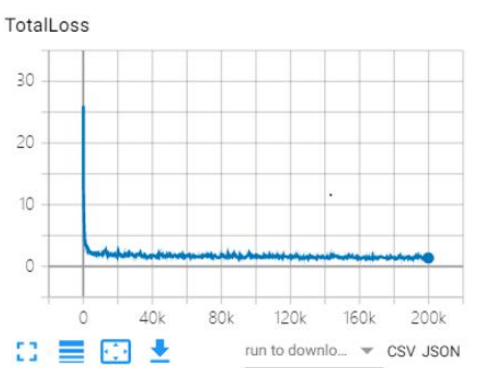

c) Total loss

Figure 9. Loss function of hand gesture recognition model in color background

The curves of classification loss, localization loss and total loss function of the two hand gesture recognition model with the number of iterations in the depth background are shown in Figure 10 a), b) and C) respectively. It can be seen from the figure that the classification loss of the model quickly converges from 21.98 to the vicinity of 1 when iterating to about 1000 steps; the location loss converges from 2.578 to the vicinity of 0.1 and oscillates nearby when iterating to about 5000 steps; the target loss of the model quickly converges from 24.9 to the vicinity of 1 and maintains oscillation when iterating to about 2000 steps.

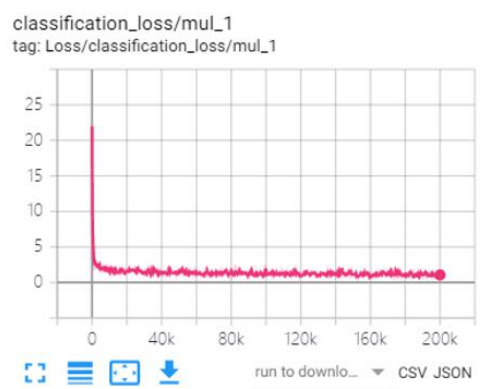

a) Classification loss

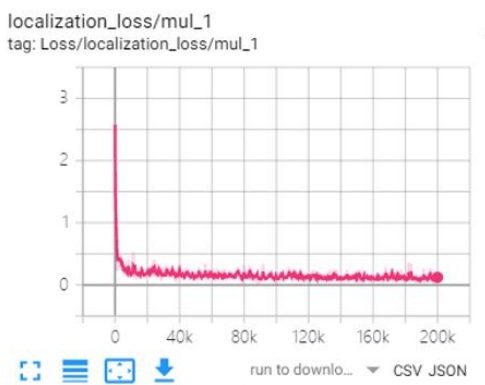

b) Localization $\cdot$ loss

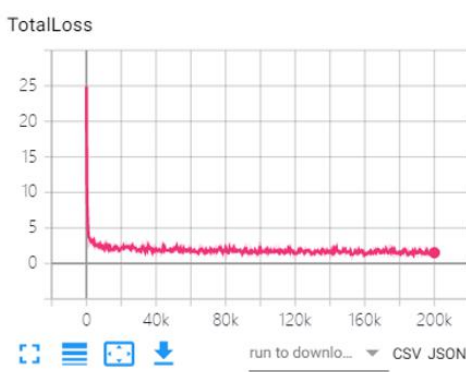

c) Total loss

Figure 10. Loss function of hand gesture recognition model in depth background

The curves of classification loss, localization loss and total loss function of the hand gesture recognition model with the number of iterations after the fusion of color map and depth map are shown in Figure 11 a), b) and c) respectively. It can be seen from the figure that through iteration, the classification loss of the model converges rapidly from 23.55 to the vicinity of 1 ; the position loss function of the model converges rapidly from 3.519 to the vicinity of 0.1 and oscillates nearby; the target loss of the model converges rapidly from 27.69 to the vicinity of 1 and keeps convergence. 


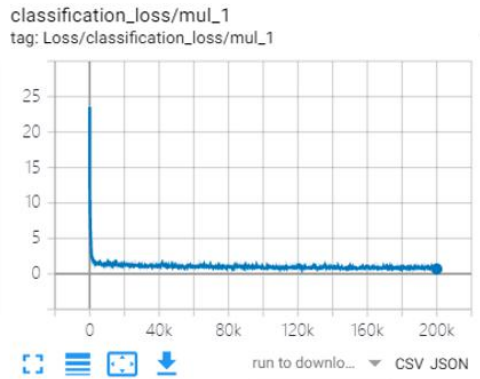

a) Classification loss

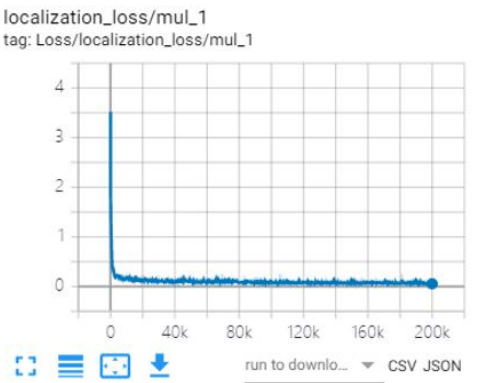

b) Localization $\cdot$ loss

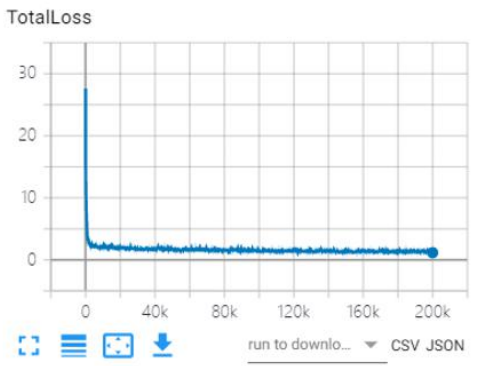

c) Total $\cdot$ loss

Figure 11. Loss function of two hand gesture recognition model with color depth fusion

(3) Average accuracy (AP)

The average accuracy is an important index to measure the accuracy of model recognition, and the average accuracy (AP) takes both precision and recall into account. Using the test set of self built database, the color background, depth background and color and depth fusion gesture recognition model are tested respectively, as shown in fig $12, a), b), c)$, d) and e) are respectively the average accuracy and the overall average accuracy (mAP) of the four defined gestures detected by the two hand self-occlusion gesture recognition model in color background.

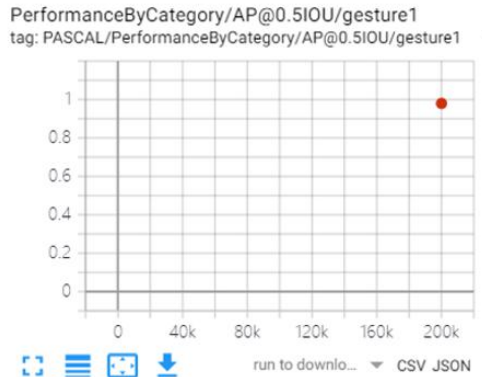

a) Average accuracy of gesture 1
PerformanceByCategory/AP@0.510U/gesture2 tag: PASCAL/PerformanceByCategory/AP@0.510U/gesture2

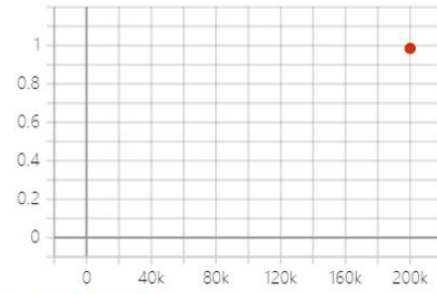

I $\equiv$

b) Average accuracy of gesture2

Precision/mAP@u.viu

tag: PASCAL/Precision

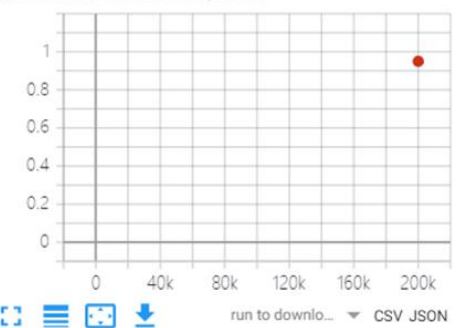

e) Overall average accuracy
PerformanceByCategory/AP@0.510U/gesture3 ag: PASCAL/PerformanceByCategory/AP@0.510U/gesture3

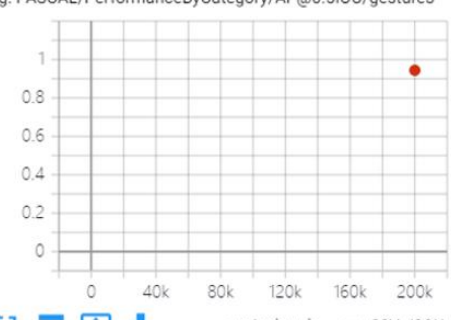

[I $\equiv \pm$ run to downio...

c) Average accuracy of gesture 3

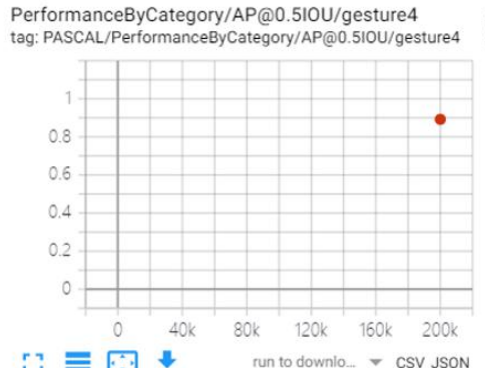

[? $\equiv$

d) Average accuracy of gesture4

Figure 12. Average accuracy of self-occlusion gesture recognition model detection in color background

In the color background, the specific values of the average accuracy of SSD based two hand gesture recognition model are shown in Table 1. In the color background, the recognition rate of four kinds of self-occlusion gestures is higher than $90 \%$, 
gesture 2 has the highest recognition rate, reaching $96.49 \%$, gesture 4 has the lowest recognition rate, the recognition rate is $90.21 \%$, and the overall average recognition rate of four kinds of gestures is $94.01 \%$.

Table 1. Detection accuracy of each category of hand gesture recognition model in color background

\begin{tabular}{ccc}
\hline & Iteration steps & Average accuracy (AP)\% \\
\hline Gesture1 & $200 \mathrm{k}$ & 95.98 \\
Gesture2 & $200 \mathrm{k}$ & 96.49 \\
Gesture3 & $200 \mathrm{k}$ & 94.36 \\
Gesture4 & $200 \mathrm{k}$ & 89.21 \\
Total & $200 \mathrm{k}$ & 93.01
\end{tabular}

In the depth background, the test set of depth map is used to test the two hand gesture recognition model in the depth background. In Figure 13, a), b), c), d) and e) are the average accuracy of four hand gesture recognition models in the depth background and the average accuracy of four hand gestures (map).

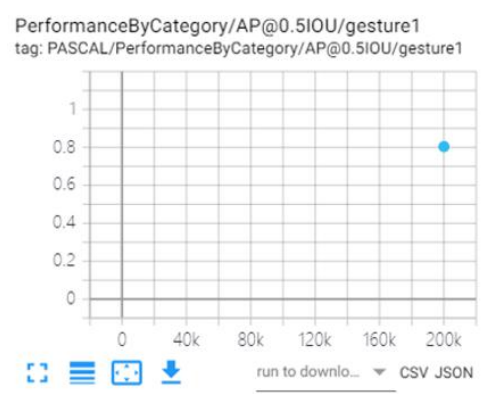

a) Average accuracy of gesture1

PerformanceByCategory/AP@0.510U/gesture4 tag: PASCAL/PerformanceByCategory/AP@0.5IOU/gesture4

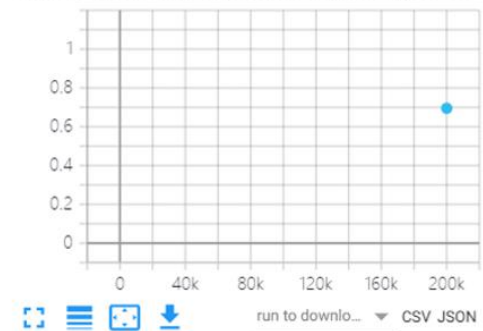

d) Average accuracy of gesture 4

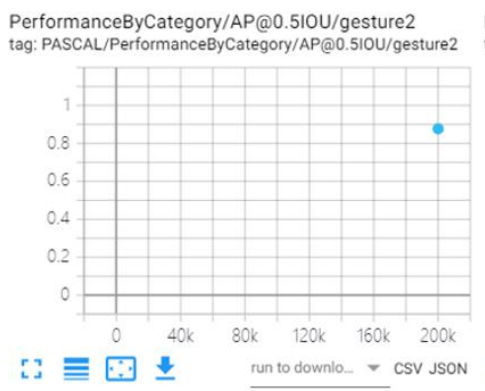

b) Average accuracy of gesture2
PerformanceByCategory/AP@0.510U/gesture3 PerformanceByCategory/AP@0.510U/gesture3

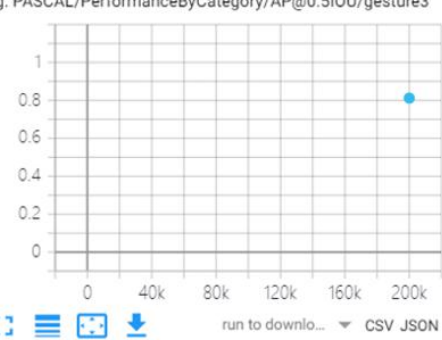

c) Average accuracy of gesture 3

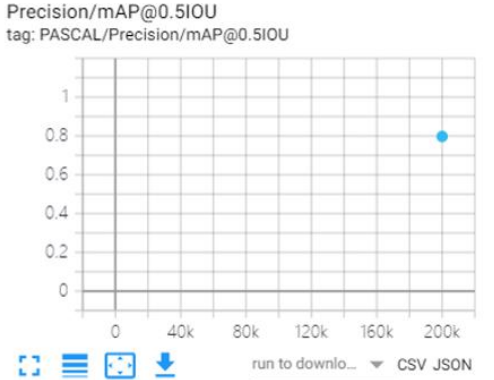

e) Overall average accuracy

Figure 13. Average accuracy of hand gesture recognition model detection in depth background

In the depth background, the specific values of the average accuracy of the two hand gesture recognition model are shown in Table 2. In the depth background, gesture 2 has the highest recognition rate, with an average recognition rate of $87.74 \%$. Gesture 4 has the lowest recognition rate, with an average recognition rate of $69.48 \%$. The overall average recognition rate of the four gestures in the depth background is $79.71 \%$. Compared with the two hand gesture recognition rate in the color 
background and the depth background, the gesture recognition rate in the color background is significantly higher than that in the depth background, which shows that the color background contains more feature information than the depth background. And gesture 4 has the lowest recognition rate in color and depth background ${ }^{[57-58]}$. Through the analysis of the experimental process, the main reason is that gesture4 has more serious self-occlusion and less feature information than the other three gestures.

Table 2. Detection accuracy of each category of hand gesture recognition model in depth background

\begin{tabular}{ccc}
\hline & Iteration steps & Average accuracy (AP)\% \\
\hline Gesture1 & $200 \mathrm{k}$ & 80.42 \\
Gesture2 & $200 \mathrm{k}$ & 87.74 \\
Gesture3 & $200 \mathrm{k}$ & 81.22 \\
Gesture4 & $200 \mathrm{k}$ & 69.48 \\
Total & $200 \mathrm{k}$ & 79.71
\end{tabular}

After the fusion of color and depth map, through the training of the fused data, the two hand self-occlusion gesture recognition model is tested. In Figure 14, a), b), c), d) and e) are respectively the average accuracy and the overall average accuracy (map) of the two hand gesture recognition model after the fusion of color and depth map.

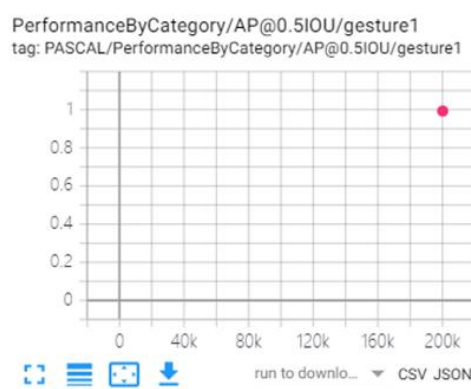

a) Average accuracy of gesture 1
PerformanceByCategory/AP@0.510U/gesture2 tag: PASCAL/PerformanceByCategory/AP@0.5IOU/gesture2

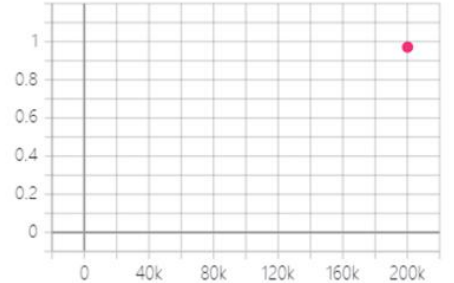

$\mathrm{U} \equiv \underline{\mathrm{\theta}}$

b) Average accuracy of gesture 2

Precision/mAP@0.510U tag: PASCAL/Precision/mAP@0.510u

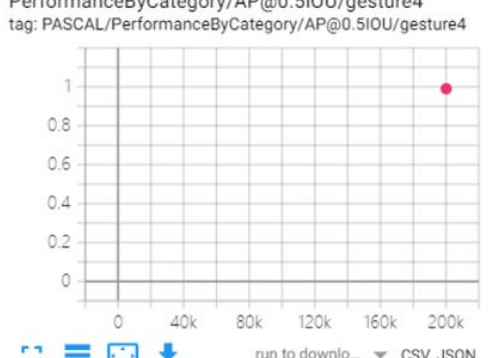

: $\equiv$

d) Average accuracy of gesture 4
PerformanceByCategory/AP@0.5IOU/gesture3 tag: PASCAL/PerformanceByCategory/AP@0.510U/gesture3

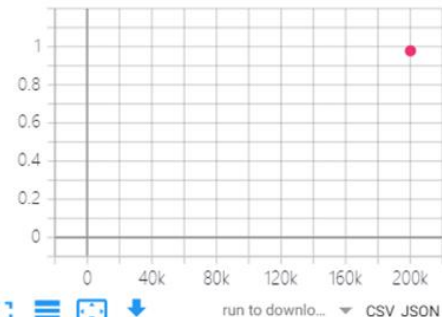

c) Average accuracy of gesture 3

Figure 14. Average accuracy of hand gesture recognition model detection under color depth fusion

After color and depth fusion, the specific values of the average accuracy of gesture recognition model detection are shown in Table 3. In the self-occlusion gesture 
recognition model under color depth fusion, gesture 2 has the highest recognition rate, with an average recognition rate of $98.11 \%$, gesture 4 has the lowest recognition rate, with an average recognition rate of $96.01 \%$. The overall average recognition rate of the four gestures under color depth fusion is as follows: $97.29 \%$.

Table 3. Detection accuracy of each category of hand gesture recognition model under color depth fusion

\begin{tabular}{ccc}
\hline & Iteration steps & Average accuracy (AP)\% \\
\hline Gesture1 & $200 \mathrm{k}$ & 97.26 \\
Gesture2 & $200 \mathrm{k}$ & 98.11 \\
Gesture3 & $200 \mathrm{k}$ & 97.78 \\
Gesture4 & $200 \mathrm{k}$ & 96.01 \\
Total & $200 \mathrm{k}$ & 97.29
\end{tabular}

Figure 15 is a histogram of the average recognition accuracy of the hand gesture recognition model in color background, depth background and color and depth fusion. It can be clearly seen from the graph that the average recognition rate of each hand gesture in depth background is lower than that in color background. The average recognition accuracy of gesture 1 is $1.28 \%$ higher than that of color background, gesture 2 is $1.62 \%$ higher than that of color background, gesture 3 is $3.42 \%$ higher than that of color background, gesture 4 is $6.8 \%$ higher than that of color background The average recognition accuracy is $4.28 \%$ higher than that of color background, so the recognition effect of color depth fusion is better than that of single depth background or color background.

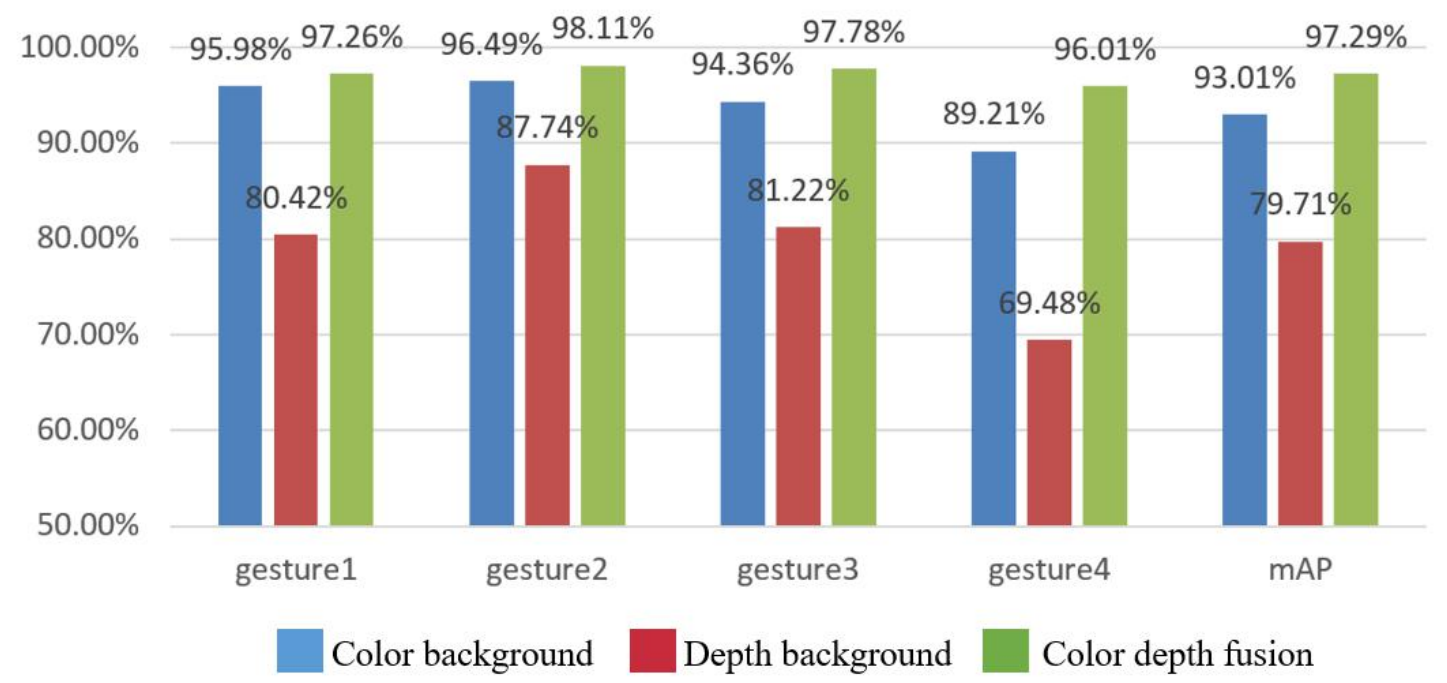

Figure 15. Average recognition accuracy of gesture types of three gesture recognition models

5.3.2 Result and analysis of gesture recognition model for object occlusion

(1) Learning rate

The gesture recognition model of occluded objects is also trained by the improved Mobilenets-SSD network, so the gesture recognition model in different 
background uses the same parameter settings. By using the learning rate decay mechanism, the initial learning rate is also set as 0.004. With continuous iterations, the learning rate decay is $95 \%$ per 30000 iterations, and the total number of training steps is 200000 . The change curve of learning rate with the number of iterations in the whole training process is shown in figure 16.

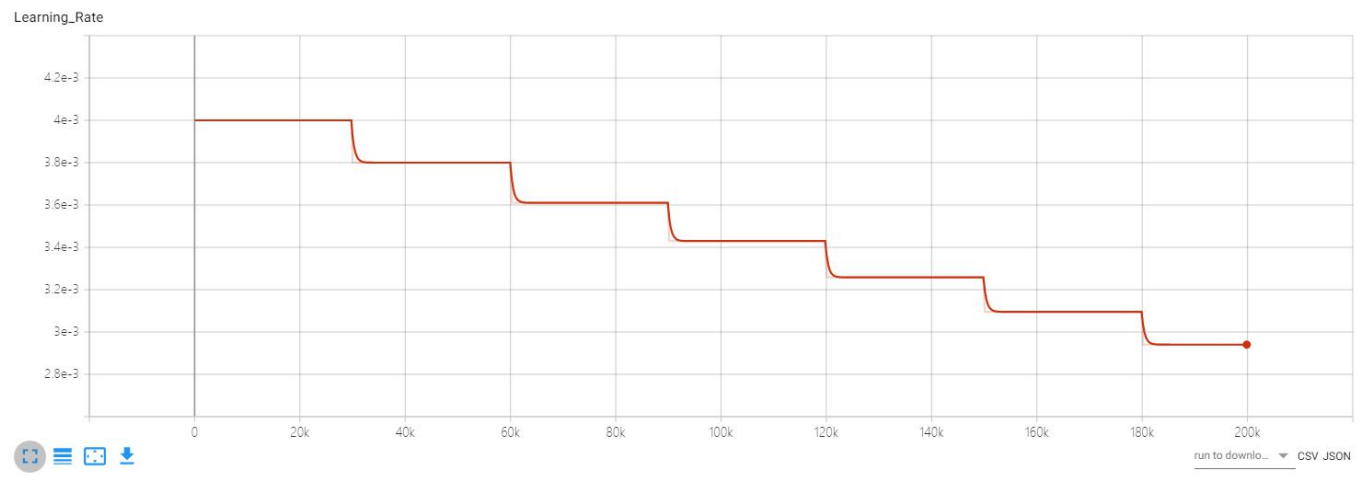

Figure 16. Change curve of learning rate

(2) Loss function

The occlusion gesture recognition model and the self-occlusion gesture model adopt the same network structure. The calculation of the loss function is the same as that of the two hand self-occlusion gesture recognition model. In figure 17, a), b) and C) are the classification loss function, the position loss function and the total target loss function of the occlusion gesture recognition model with the change curve of the number of iterations. It can be seen from the figure that all kinds of loss functions of occlusion gesture recognition model in color background converge rapidly and oscillate near 0 , which shows that the model has good robustness.

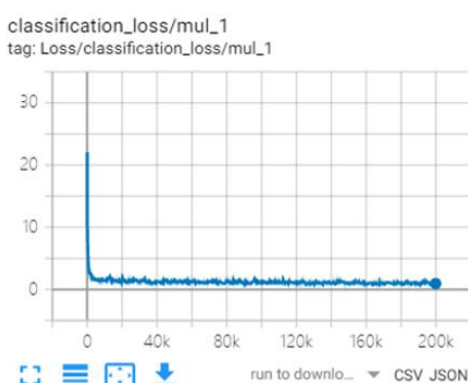

a) Classification loss

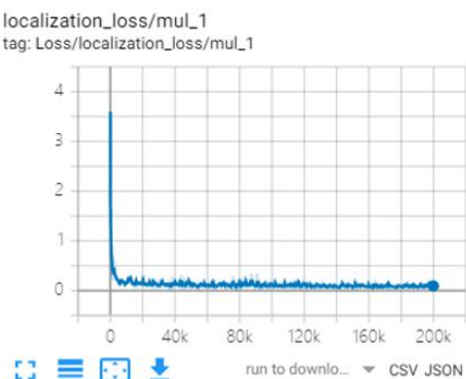

b) Localization loss

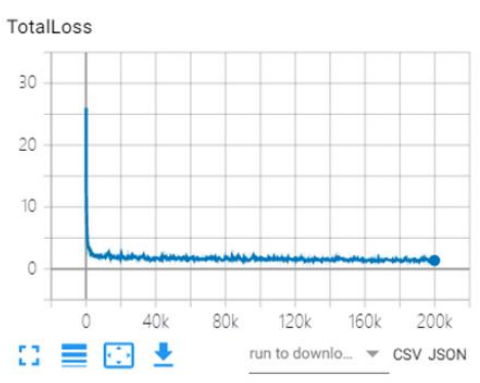

c) Total loss

Figure 17. Loss function of occlusion gesture recognition model in color background

Under the depth background, the classification loss, position loss and target total loss functions of occlusion gesture recognition model change with the number of iterations as shown in figure $18 \mathrm{a}$ ), b) and c). It can be seen from the figure that when the classification loss is iterated to about 20000 times, the loss function value drops from 3.1 to near 1 and oscillates near 1; when the location loss is iterated to about 20000 times, the loss function value drops from 0.62 to 
near 0.1 and oscillates near 0.1 , basically reaching convergence; when the target loss of the model is iterated to about 20000 steps, it drops from 4 to near 1.5 and oscillates near 1.5 Swing, basically convergence.

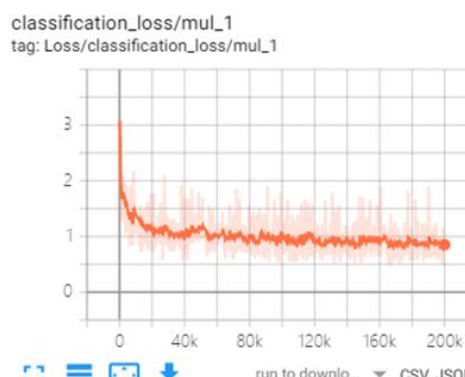

[? $\equiv$

a) Classification loss

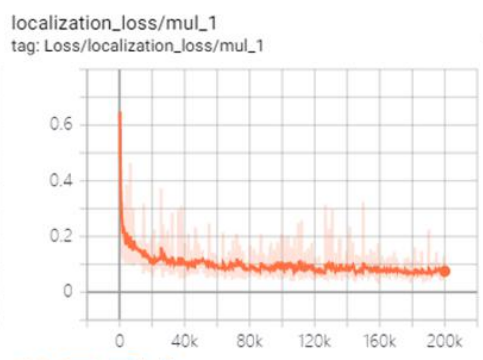

[u

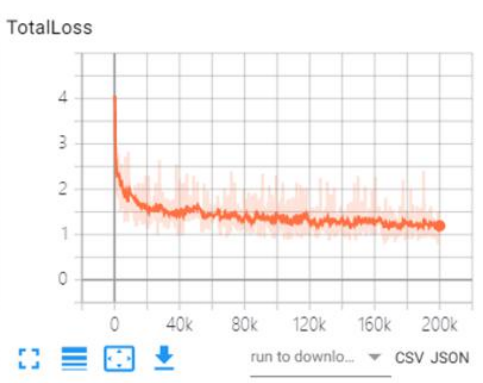

c) Total loss

Figure 18. Loss function of occlusion gesture recognition model in depth background

In figure $19 \mathrm{a}), \mathrm{b}$ ) and c) are the classification loss, position loss and total loss function of the object occlusion gesture recognition model with the number of iterations after color depth fusion. It can be seen from the figure that the classification loss of the iterative model converges rapidly from 9.2 to the vicinity of 1 and keeps convergence; the location loss of the model converges rapidly from 2.3 to the vicinity of 0.1 and keeps convergence; the overall target loss of the model converges rapidly from 12 to the vicinity of 2 . It can be seen from the convergence of all kinds of loss functions in the figure that all kinds of loss functions of the model converge rapidly, indicating that the training effect of the model is good.

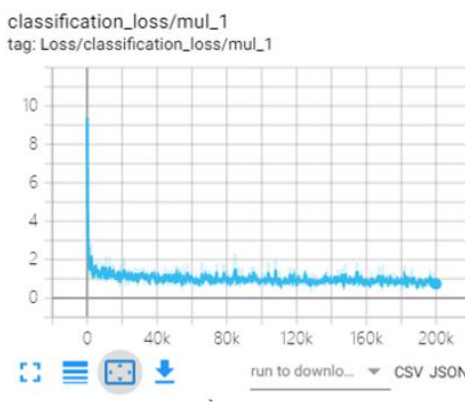

a) Classification loss

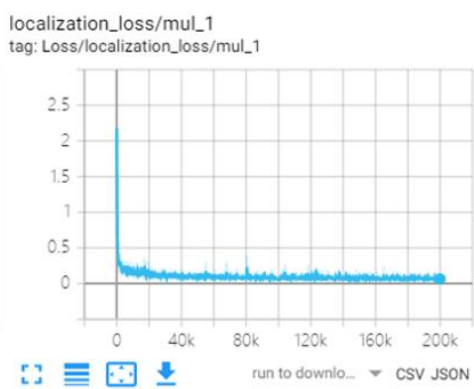

b) Localization loss

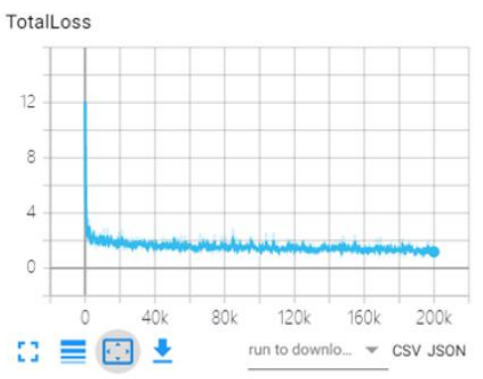

c) Total loss

Figure 19. Loss function of occlusion gesture recognition model after color depth fusion

(3) Average accuracy (AP)

In the color background, the trained model is tested by using the self built database of object occlusion gesture. In figure 20 a), b), c), d) and e) are the average detection accuracy and overall average accuracy of gesture recognition model for five kinds of occlusion gestures in the color background. 


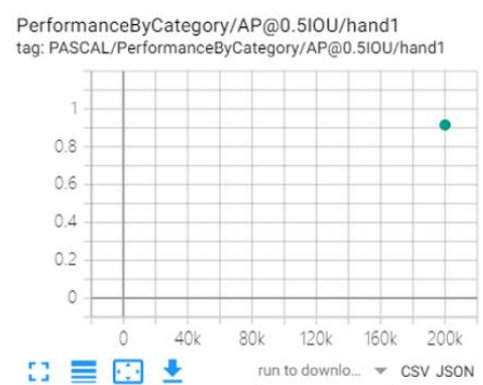

a) Average accuracy of hand 1

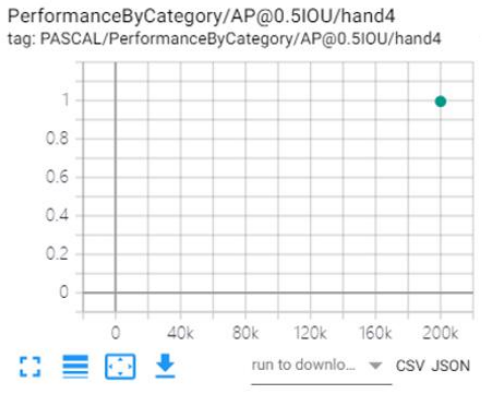

d) Average accuracy of hand 4
PerformanceByCategory/AP@0.510U/hand2 tag: PASCAL/PerformanceByCategory/AP@0.510U/hand2

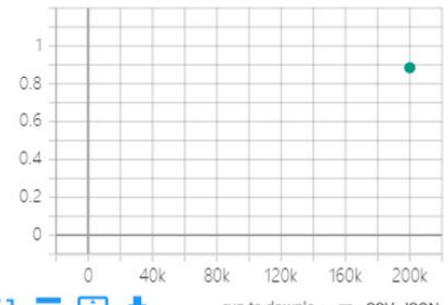

b) Average accuracy of hand 2

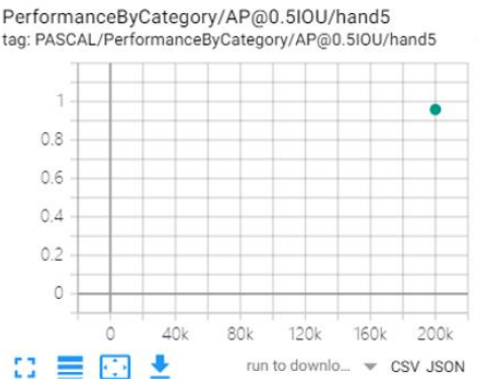

e) Average accuracy of hand 5

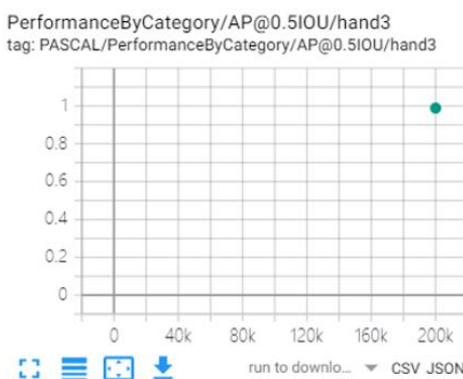

c) Average accuracy of hand 3

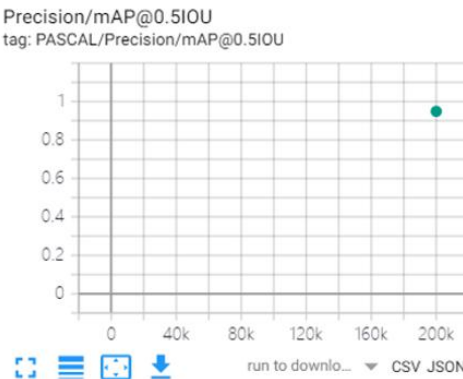

f) Overall average accuracy

Figure 20. Average accuracy of occlusion gesture recognition model detection in color background

The specific values of the average accuracy of the occluded gesture recognition model in the color background are shown in table 4. Among the five gestures, Hand 2 has the lowest gesture recognition rate, only $88.5 \%$. The other four occluded gestures have gesture recognition rates above $90 \%$. The overall average recognition rate of the five gestures is $92.95 \%$.

Table 4. Detection accuracy of various categor ies of object occlusion gesture recognition model in color background

\begin{tabular}{ccc}
\hline & Iteration steps & Average accuracy (AP) \% \\
\hline Hand1 & $200 \mathrm{k}$ & 91.55 \\
Hand2 & $200 \mathrm{k}$ & 88.50 \\
Hand3 & $200 \mathrm{k}$ & 94.39 \\
Hand4 & $200 \mathrm{k}$ & 94.32 \\
Hand5 & $200 \mathrm{k}$ & 93.48 \\
Total & $200 \mathrm{k}$ & 92.95
\end{tabular}

In the depth background, the test set of the depth map is used to test the object occlusion gesture recognition model in the depth background, and the tensorboard is also used to visualize one of the average accuracy indicators. In figure $21 \mathrm{a}$ ), b), c), d) and e) are respectively the average accuracy of the object occlusion gesture recognition model in the depth background and the average accuracy of the five gestures (map). 


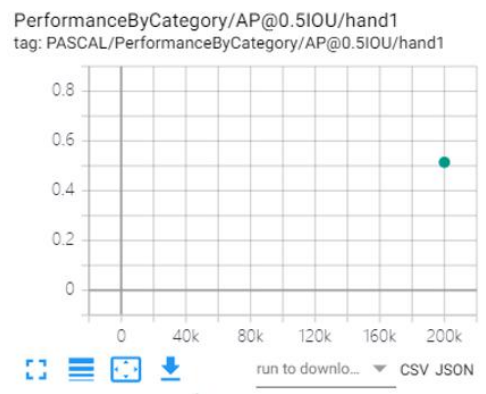

a) Average accuracy of hand1

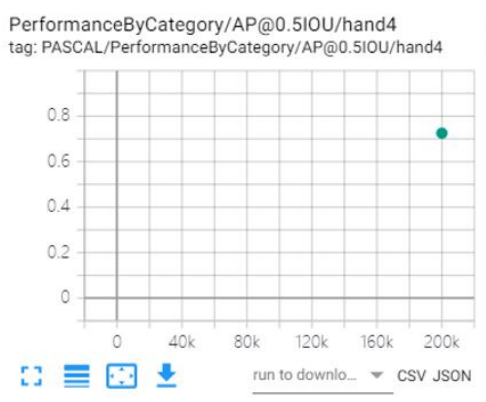

d) Average accuracy of hand 4

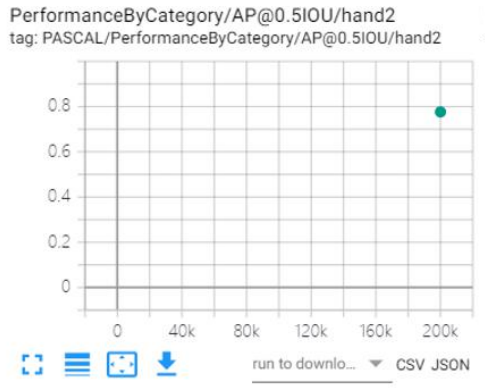

b) Average accuracy of hand 2

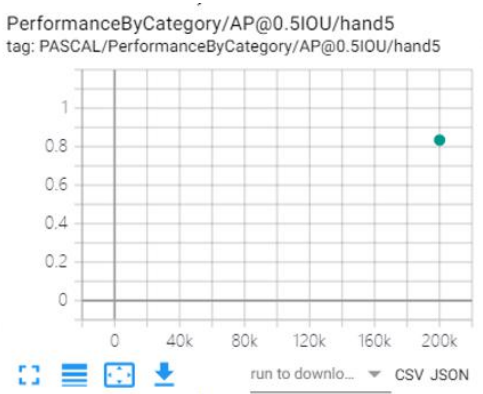

e) Average accuracy of hand 5

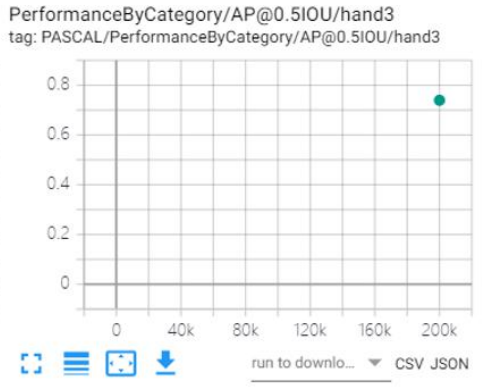

c) Average accuracy of hand 3

Figure 21. Average accuracy of gesture recognition model for occlusion in depth background

Under the depth background, the specific values of the average accuracy of the object occlusion gesture recognition model are shown in Table 5. Under the depth background, the highest recognition rate of the occlusion gesture recognition model is hand5, the average accuracy is $83.43 \%$, the lowest recognition rate is Hand1, the average accuracy is $53.44 \%$, and the overall average accuracy of the five gestures under the depth background is $72.19 \%$. Compared with the recognition rate of object occlusion gesture in color background and depth background, the recognition rate in depth background is far lower than that in color background, which shows that the gesture in depth background contains far less feature information than that in color background.

Table 5. Detection accuracy of various categor ies of object occlusion gesture recognition model in depth background

\begin{tabular}{ccc}
\hline & Iteration steps & Average accuracy (AP)\% \\
\hline Hand1 & $200 \mathrm{k}$ & 53.44 \\
Hand2 & $200 \mathrm{k}$ & 77.64 \\
Hand3 & $200 \mathrm{k}$ & 73.95 \\
Hand4 & $200 \mathrm{k}$ & 72.51 \\
Hand5 & $200 \mathrm{k}$ & 83.43 \\
\hline
\end{tabular}


In the case of color depth fusion, the model is tested by the test set after fusion, and one of the average precision indexes is visualized by Tensorboard. In figure $22 \mathrm{a}), \mathrm{b}), \mathrm{c}), \mathrm{d})$ and e) are the average precision of five gestures detected by the object occlusion gesture recognition model under color depth fusion and the average precision of five gestures (map).

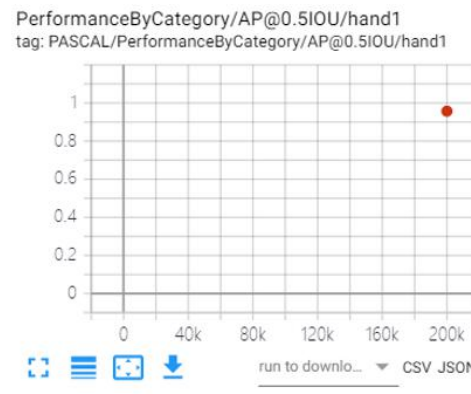

a) Average accuracy of hand 1

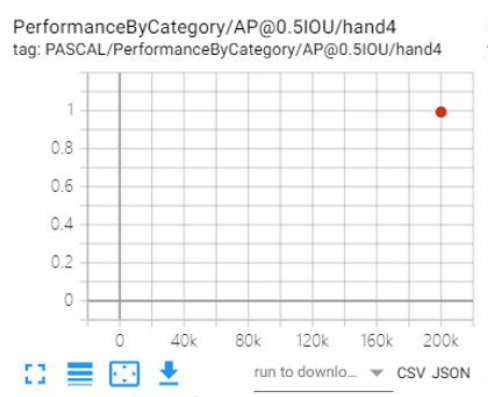

d) Average accuracy of hand 4

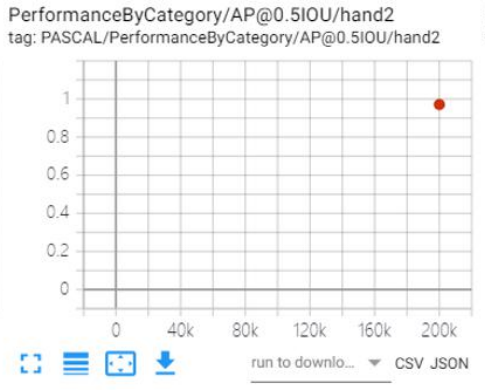

b) Average accuracy of hand 2

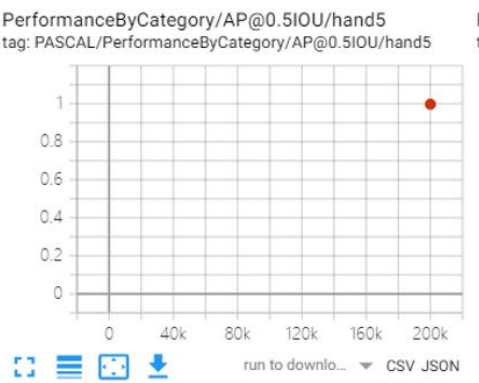

e) Average accuracy of hand 5

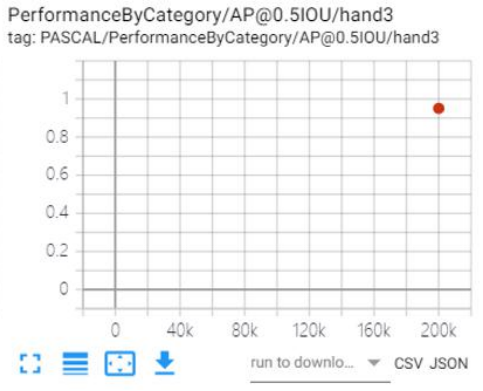

c) Average accuracy of hand 3

Figure 22. Average accuracy of occlusion gesture recognition model under color depth fusion

After color depth image fusion, the specific values of the detection average accuracy of the occlusion gesture recognition model are shown in Table 6 . In the gesture recognition model of color depth fusion, the highest gesture recognition rate is hand5, the average recognition rate is $98.71 \%$, the lowest gesture 
recognition rate is hand3, the average recognition rate is $94.79 \%$, and the overall average recognition accuracy of the five types of gestures is $96.49 \%$.

Table 6. Detection accuracy of various categor ies of object occlusion gesture recognition model under color depth fusion

\begin{tabular}{ccc}
\hline & Iteration steps & Average accuracy (AP)\% \\
\hline Hand1 & $200 \mathrm{k}$ & 96.52 \\
Hand2 & $200 \mathrm{k}$ & 96.23 \\
Hand3 & $200 \mathrm{k}$ & 95.11 \\
Hand4 & $200 \mathrm{k}$ & 97.30 \\
Hand5 & $200 \mathrm{k}$ & 98.71 \\
Total & $200 \mathrm{k}$ & 96.49 \\
\hline
\end{tabular}

The histogram of the average recognition accuracy of the object occlusion gesture recognition model in the color background, depth background and color and depth fusion of various types of gesture is shown in figure 23. It can be seen clearly from the figure that the average recognition rate of each gesture in the depth background is far lower than that in the color background, indicating that there are more feature information in the color background than in the depth background. After the fusion of color and depth, the average recognition accuracy of hand gesture is improved compared with that of color background. The average recognition accuracy of Hand1 is increased by $4.97 \%$, Hand 2 by $7.73 \%$, and hand 3 by $0.72 \%$. The average recognition accuracy of hand 4 is increased by $2.98 \%$ and hand 5 by $5.32 \%$. The overall average recognition accuracy of the five gestures is improved by $3.54 \%$. It can be seen that the effect of gesture recognition model after color depth pre fusion is better than that of gesture recognition model in a single background.

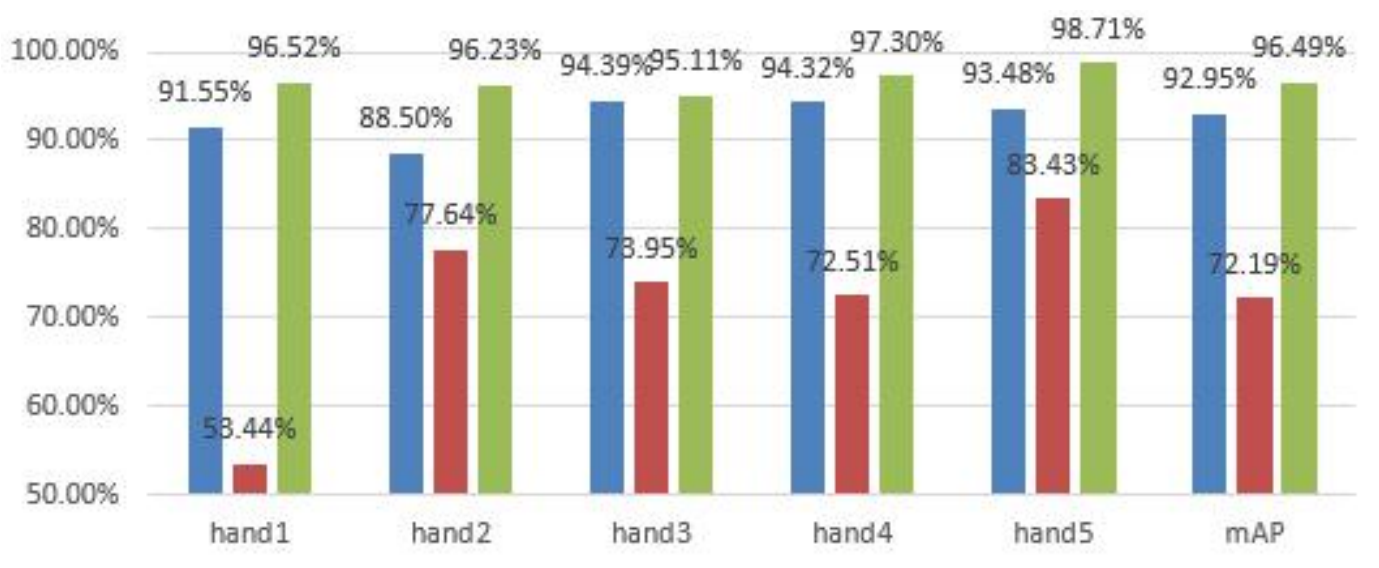

Color background $\square$ Depth background $\square$ Color depth fusion

Figure 23. Average recognition accuracy of gesture types of three gesture recognition models

\section{Summary}


In order to solve the occlusion problem in the process of gesture recognition, an occlusion gesture recognition method based on improved SSD algorithm is proposed. Collected a large number of object occlusion gestures and self-occlusion gestures respectively, and established a gesture database. Based on the established database, trained self-occlusion and object occlusion gesture recognition models based on the fusion of color maps, depth maps, and color maps and depth maps, and the results are analyzed. The experimental results show that, based on the improved occlusion gesture recognition method of the SSD network, the gesture recognition rate obtained by fusing the color map and the depth map is superior to the occlusion gesture recognition rate using the color map or the depth map alone. The improved SSD algorithm has a good effect on the recognition of occlusion gestures.

\section{Acknowledgements}

This work was supported by grants of National Natural Science Foundation of China (Grant Nos. 51575407 , 51505349 , 61733011 , 41906177 ); the Grants of Hubei Provincial Department of Education (D20191105); the Grants of National Defense Pre-Research Foundation of Wuhan University of Science and Technology (GF 201705 ) and Open Fund of the Key Laboratory for Metallurgical Equipment and Control of Ministry of Education in Wuhan University of Science and Technology (2018B07).

\section{Reference:}

[1] Traver V J, Latorre-Carmona P, Salvador-Balaguer E, Pla F, Javidi B. Three-dicmensional integral imaging for gesture recognition under occlusions. IEEE Signal Processing Letters, 2016, 24(2): 171-175.

[2] Kuznetsova A, Laura Leal-Taixé, Rosenhahn B. Real-Time Sign Language Recognition Using a Consumer Depth Camera. International Conference on Computer Vision Workshops. IEEE, 2013:83-90.

[3] Maqueda A I, Del-Blanco C R, Jaureguizar F, Garcí N. Human-Computer Interaction based on Visual Hand-Gesture Recognition using Volumetric Spatiograms of Local Binary Patterns. Computer Vision and Image Understanding, 2015, 141:126-137.

[4] Padam Priyal S, Bora P K. A robust static hand gesture recognition system using geometry based normalizations and Krawtchouk moments. Pattern Recognition, 2013, 46(8):2202-2219.

[5] Belongie S, Malik J, Puzicha J. Shape matching and object recognition using shape contexts. IEEE Transactions on Pattern Analysis \& Machine Intelligence, 2002 (4): 509-522.

[6] Dadashzadeh A, Targhi A T, Tahmasbi M, Mirmehdi M. HGR-Net: a fusion network for hand gesture segmentation and recognition. IET Computer Vision, 
2019, 13(8): 700-707.

[7] Rastgoo R, Kiani K, Escalera S. Hand sign language recognition using multi-view hand skeleton. Expert Systems with Applications, 2020: 113336.

[8] Rangesh A, Ohn-Bar E, Trivedi M M. Hidden hands: Tracking hands with an occlusion aware tracker. Proceedings of the IEEE Conference on Computer Vision and Pattern Recognition Workshops. 2016: 19-26.

[9] Poon G, Kwan K C, Pang W M. Occlusion-robust bimanual gesture recognition by fusing multi-views. Multimedia Tools and Applications, 2019, 78(16): 23469-23488.

[10]Gongfa Li, Ze Liu, Guozhang Jiang, Hegen Xiong and Honghai Liu. Numerical simulation of the influence factors for rotary kiln in temperature field and stress field and the structure optimization. Advances in Mechanical Engineering, 2015, 7(6): 1687814015589667, DOI:10.1177/ 1687814015589667.

[11]Zhihua Cui, Fei Xue, Shiqiang Zhang, Xingjuan Cai, Yang Cao, Wensheng Zhang, Jinjun Chen, A Hybrid BlockChain-Based Identity Authentication Scheme for Multi-WSN, IEEE Transactions on Services Computing, 2020, 13(2): 241-251.

[12]Wenjun Chang, Gongfa Li, Jianyi Kong, Ying Sun, Guozhang Jiang and Honghai Liu. Thermal mechanical stress analysis of ladle lining with integral brick joint. Archives of Metallurgy and Materials, 2018, 63(2):659-666.

[13]Hassan M, Rehmani M, Chen J. DEAL: Differentially Private Auction for Blockchain based Microgrids Energy Trading, IEEE Transactions on Services Computing, 2019, DOI: 10.1109/TSC.2019.2947471.

[14]M. Hassan, M. Rehmani, and J. Chen, Differential Privacy Techniques for Cyber Physical Systems: A Survey, IEEE Communications Surveys and Tutorials, 2019, DOI: 10.1109/COMST.2019.2944748.

[15] Gongfa Li, Jia Liu, Guozhang Jiang and Honghai Liu. Numerical simulation of temperature field and thermal stress field in the new type of ladle with the nanometer adiabatic material. Advances in Mechanical Engineering, 2015, 7(4): 1687814015575988 , DOI:10.1177 / 1687814015575988.

[16]Wentao Cheng, Ying Sun, Gongfa Li, Guozhang Jiang, Honghai Liu. Jointly network: a network based on CNN and RBM for gesture recognition. Neural Computing and Applications, 2019, 31(Supplement 1): 309-323, DOI:10.1007/s00521-018-3775-8.

[17]Gongfa Li, Du Jiang, Ying Sun, Guozhang Jiang and Bo Tao. Life prediction mechanism of ladle composite structure body based on simulation technology. 
Archives of Metallurgy and Materials, 2019, 64(4):1555-1562.

[18]Zhihua Cui, Jiangjiang Zhang, Di Wu, Xingjuan Cai, Hui Wang, Wensheng Zhang, Jinjun Chen, Hybrid Many-Objective Particle Swarm Optimization Algorithm for Green Coal Production Problem, Information Sciences, 2020, 518: 256-271.

[19]Jiabing Hu, Ying Sun, Gongfa Li, Guozhang Jiang and Bo Tao. Probability analysis for grasp planning facing the field of medical robotics. Measurement, 2019, 141:227-234.

[20] Sangaiah A K, Pham H, Qiu T, Muhammad K. Convergence of deep machine learning and parallel computing environment for bio - engineering applications. Concurrency and Computation: Practice and Experience, 2020, 32(1).

[21] Ying Sun, Jiabing Hu, Gongfa Li, Guozhang Jiang, Hegen Xiong, Bo Tao, Zujia Zheng, Du Jiang. Gear reducer optimal design based on computer multimedia simulation. The Journal of Supercomputing, 2020, 76(6):4132-4148.

[22]Li Huang, Qiaobo Fu, Gongfa Li, Bowen Luo, Disi Chen and Hui Yu. Improvement of maximum variance weight partitioning particle filter in urban computing and intelligence. IEEE Access, 2019, 7:106527-106535.

[23]Du Jiang, Zujia Zheng, Gongfa Li, Ying Sun, Jianyi Kong, Guozhang Jiang, Hegen Xiong, Bo Tao, Shuang Xu, Honghai Liu, Zhaojie Ju. Gesture recognition based on binocular vision. Cluster Computing, 2019, 22(Supplement 6):13261-13271 , DOI:10.1007/s10586-018-1844-5.

[24]Ruyi Ma, Leilei Zhang, Gongfa Li, Du Jiang, Shuang Xu, Disi Chen. Grasping force prediction based on sEMG signals. Alexandria Engineering Journal, 2020, 59(3):1135-1147.

[25]Jinrong Tian, Wentao Cheng, Ying Sun, Gongfa Li, Du Jiang, Guozhang Jiang, Bo Tao, Haoyi Zhao, Disi Chen. Gesture recognition based on multilevel multimodal feature fusion. Journal of Intelligent \& Fuzzy Systems, 2020, 38(3):2539-2550.

[26]Ying Sun, Jinrong Tian, Du Jiang,Bo Tao, Ying Liu, Juntong Yun, Disi Chen. Numerical simulation of thermal insulation and longevity performance in new lightweight ladle. Concurrency and Computation: Practice and Experience, 2020, DOI:10.1002/CPE.5830.

[27]Gongfa Li, Leilei Zhang, Ying Sun and Jianyi Kong. Towards the semg hand: internet of things sensors and haptic feedback application. Multimedia Tools and Applications, 2019, 78(21):29765-29782. 
[28] Ying Sun, Chao Xu, Gongfa Li, Wanfen Xu, Jianyi Kong, Du Jiang, Bo Tao, Disi Chen. Intelligent human computer interaction based on non redundant EMG signal. Alexandria Engineering Journal, 2020, 59(3):1149-1157.

[29]Ying Sun, Cuiqiao Li, Gongfa Li, Guozhang Jiang, Du Jiang, Honghai Liu, Zhigao Zheng, Wanneng Shu. Gesture recognition based on kinect and sEMG signal fusion. Mobile Networks and Applications, 2018, 23(4):797-805.

[30] Shangchun Liao, Gongfa Li, Jiahan Li, Du Jiang, Guozhang Jiang, Ying Sun, Bo Tao, Haoyi Zhao, Disi Chen. Multi-object intergroup gesture recognition combined with fusion feature and KNN algorithm. Journal of Intelligent \& Fuzzy Systems, 2020, 38(3):2725-2735.

[31]Gongfa Li, Jiahan Li, Zhaojie Ju, Ying Sun and Jianyi Kong. A novel feature extraction method for machine learning based on surface electromyography from healthy brain. Neural Computing \& Applications, 2019, 31(12): 9013-9022.

[32][45]Tinggui Chen, Shiwen Wu, Jianjun Yang, Guodong Cong, Gongfa Li. Modeling of Emergency Supply Scheduling Problem Based on Reliability and Its Solution Algorithm under Variable Road Network after Sudden-Onset Disasters[J]. Complexity, 2020:7501891.

[33]Du Jiang, Gongfa Li, Ying Sun, Jianyi Kong and Bo Tao. Gesture recognition based on skeletonization algorithm and cnn with asl database. Multimedia Tools and Applications, 2019, 78(21), 29953-29970.

[34]Tinggui Chen, Shiwen Wu, Jianjun Yang and Guodong Cong. Risk Propagation Model and Its Simulation of Emergency Logistics Network Based on Material Reliability. International Journal of Environmental Research and Public Health, 2019, 16(23), 4677.

[35]Gongfa Li, Du Jiang, Yanling Zhou, Guozhang Jiang, Jianyi Kong and Gunasekaran Manogaran. Human lesion detection method based on image information and brain signal. IEEE Access, 2019, 7:11533-11542.

[36]Tinggui Chen, Qianqian Li, Jianjun Yang, Guodong Cong and Gongfa Li. Modeling of the Public Opinion Polarization Process with the Considerations of Individual Heterogeneity and Dynamic Conformity. Mathematics, 2019, 7(10), 917.

[37]He M. Distinguish computer generated and digital images: A CNN solution. Concurrency and computation: practice and experience, 2019, 31(12):e4788.1-e4788.10.

[38]Gongfa Li, Hao Wu, Guozhang Jiang, Shuang Xu and Honghai Liu. Dynamic 
gesture recognition in the internet of things. IEEE Access, 2019, 7, 23713-23724.

[39]Jinxian Qi, Guozhang Jiang, Gongfa Li, Ying Sun and Bo Tao. Intelligent human-computer interaction based on surface emg gesture recognition. IEEE Access, 2019, 7: 61378-61387.

[40]Tinggui Chen, Qianqian Li, Peihua Fu, Jianjun Yang, Chonghuan Xu, Guodong Cong, Gongfa Li. Public Opinion Polarization by Individual Revenue from the Social Preference Theory[J]. International Journal of Environmental Research and Public Health, 2020, 17(3), 946.

[41]Gongfa Li, Heng Tang, Ying Sun, Jianyi Kong, Guozhang Jiang, Du Jiang, Bo Tao, Shuang $\mathrm{Xu}$, Honghai Liu. Hand gesture recognition based on convolution neural network. Cluster Computing, 2019, 22(Supplement 2): 2719-2729, DOI:10.1007/s10586-017-1435-x.

[42]Xingjuan Cai, Zhaoming Hu, Peng Zhao, WenSheng Zhang and Jinjun Chen, A hybrid recommendation system with many-objective evolutionary algorithm, Expert Systems With Applications, 2020, 159,113648.

[43]Xingjuan Cai, Zhaoming $\mathrm{Hu}$, Jinjun Chen, A many-objective optimization recommendation algorithm based on knowledge mining, Information Sciences, 2020, 537:148-161.

[44]Xingjuan Cai, Penghong Wang, Yuan Niu, Zhihua Cui, Wensheng Zhang and Jinjun Chen, Weight convergence analysis of DV-hop localization algorithm with GA[J], Soft Computing, 2020, DOI: 10.1007/s00500-020-05088-z.

[45]Chengcheng Li, Gongfa Li, Guozhang Li, Disi Chen and Honghai Liu. Surface emg data aggregation processing for intelligent prosthetic action recognition. Neural Computing \& Applications, 2018, DOI:10.1007/s0 0521-018 -3909-z.

[46]Chong Tan, Ying Sun, Gongfa Li, Guozhang Jiang, Disi Chen and Honghai Liu. Research on gesture recognition of smart data fusion features in the IoT. Neural Computing \& Applications, 2019, DOI:10.1007/s 00521-019- 04023-0.

[47]Mingchao Yu, Gongfa Li, Du Jiang, Guozhang Jiang, Bo Tao and Disi Chen. Hand medical monitoring system based on machine learning and optimal EMG feature set . Personal and Ubiquitous Computing, 2019, DOI:10.1007/s 00779-019 -01285-2.

[48]Bowen Luo, Ying Sun, Gongfa Li, Disi Chen, Zhaojie Ju. Decomposition algorithm for depth image of human health posture based on brain health. Neural Computing and Applications, 2020, 32(10):6327-6342.

[49]Jinxian Qi, Guozhang Jiang, Gongfa Li, Ying Sun, Bo Tao. Surface EMG hand gesture recognition system based on PCA and GRNN. Neural Computing and 
Applications, 2020, 32(10):6343-6351.

[50]Mingchao Yu, Gongfa Li, Du Jiang, Guozhang Jiang, Fei Zeng, Haoyi Zhao, Disi Chen. Application of PSO-RBF neural network in gesture recognition of continuous surface EMG signals. Journal of Intelligent \& Fuzzy Systems, 2020, 38(3):2469-2480.

[51]Bei Li, Ying Sun, Gongfa Li, Jianyi Kong, Guozhang Jiang, Du Jiang, Bo Tao, Shuang $\mathrm{Xu}$, Honghai Liu. Gesture recognition based on modified adaptive orthogonal matching pursuit algorithm. Cluster Computing, 2019, 22 (Supplement 1):503-512, DOI:10.1007/s10586-017-1231-7.

[52]Du Jiang, Gongfa Li, Ying Sun, Jianyi Kong, Bo Tao and Disi Chen. Grip strength forecast and rehabilitative guidance based on adaptive neural fuzzy inference system using sEMG . Personal and Ubiquitous Computing, 2019, DOI:10.1007/s 00779 -019-01268-3.

[53] Yangwei Cheng, Gongfa Li, Jiahan Li, Ying Sun, Guozhang Jiang, Fei Zeng, Haoyi Zhao, Disi Chen. Visualization of activated muscle area based on sEMG. Journal of Intelligent \& Fuzzy Systems, 2020, 38(3):2623-2634.

[54]Gongfa Li, Peixin Qu, Jianyi Kong, et al. Influence of working lining parameters on temperature and stress field of ladle. Applied Mathematics \& Information Sciences, 2013, 7(2):439-448.

[55]Yang He, Gongfa Li, Yajie Liao, Ying Sun, Jianyi Kong, Guozhang Jiang, Du Jiang, Honghai Liu. Gesture recognition based on an improved local sparse representation classification algorithm. Cluster Computing, 2019, 22(Supplement 5):10935-10946, DOI:10.1007/s10586-017-1237-1.

[56] Yaoqing Weng, Ying Sun, Du Jiang, Bo Tao, Ying Liu, Juntong Yun, Dalin Zhou .Enhancement of real-time grasp detection by cascaded deep convolutional neural networks.Concurrency and Computation: Practice and Experience, 2020, DOI:10.1002/CPE.5976.

[57]Xiaowu Chen, Guozhang Jiang, Le Yang, Gongfa Li, Feng Xiang. Redesign of enterprise lean production system based on environmental dynamism. Concurrency and Computation: Practice and Experience, 2020, 32(14):e5706.

[58]Xu Zheng, Q Zhou. Special Issue on Applications and Techniques in Cyber Intelligence Concurrency and Computation: Practice and Experience. Concurrency and Computation: Practice and Experience, 2020, 32(6). 